\title{
Improving the performance of univariate control charts for abnormal detection and classification
}

Christos Yiakopoulos ${ }^{\mathrm{a}}$, Maria Koutsoudaki ${ }^{\mathrm{a}}$, Konstantinos Gryllias ${ }^{\mathrm{b}, \mathrm{c}}$, Ioannis Antoniadis ${ }^{\mathrm{a}}$

${ }^{a}$ Dynamics and Structures Laboratory, Machine Design and Control Systems Section, School of Mechanical Engineering, National Technical University of Athens, Athens, Greece

${ }^{\mathrm{b}}$ KU Leuven, Department of Mechanical Engineering, Division PMA, Celestijnenlaan 300, BOX 2420, Leuven, 3001, Belgium

${ }^{\mathrm{c}}$ Member of Flanders Make

\begin{abstract}
Bearing failures in rotating machinery can cause machine breakdown and economical loss, if no effective actions are taken on time. Therefore, it is of prime importance to detect accurately the presence of faults, especially at their early stage, to prevent sequent damage and reduce costly downtime. The machinery fault diagnosis follows a roadmap of data acquisition, feature extraction and diagnostic decision making, in which mechanical vibration fault feature extraction is the foundation and the key to obtain an accurate diagnostic result. A challenge in this area is the selection of the most sensitive features for various types of fault, especially when the characteristics of failures are difficult to be extracted. Thus, a plethora of complex data-driven fault diagnosis methods are fed by prominent features, which are extracted and reduced through traditional or modern algorithms. Since most of the available datasets are captured during normal operating conditions, the last decade a number of novelty detection methods, able to work when only normal data are available, have been developed. In this study, a hybrid method combining univariate control charts and a feature extraction scheme is introduced focusing towards an abnormal change detection and classification, under the assumption that measurements under normal operating conditions of the machinery are available. The feature extraction method integrates the morphological operators and the Morlet wavelets. The effectiveness of the proposed methodology is validated on two different experimental cases with bearing faults, demonstrating that the proposed approach can improve the fault detection and classification performance of conventional control charts.
\end{abstract}

\section{Keywords}

Novelty detection, bearing fault diagnosis, feature extraction, control charts, morphological gradients, Morlet wavelet

\begin{tabular}{|llll|}
\hline \multicolumn{2}{ll}{ Abbreviations } & SE & Structuring Element \\
SNR & Signal-Noise Ratio & CL & Centre Line \\
SVD & Singular Value Decomposition & LCL & Lower Control Limit \\
SV & Singular Value & UCL & Upper Control Limit \\
FC & Center Frequency & KUC & Kurtosis Criterion \\
CSMW & Complex Shifted Morlet wavelet & FFT & Fast Fourier Transform \\
EWMA & Exponentially Weight Moving Average & IFFT & Inverse Fast Fourier Transform \\
MSPC & Multivariate Statistical Process Control & SPC & CUmulative SUM \\
MM & Mathematical Morphology & OR & Statistical Process Control \\
MO & Morphological Operator & IR & Inner Race \\
BG & Beucher Gradient & MSV & Mean Singular Value \\
\hline
\end{tabular}




\begin{tabular}{|llll|}
\hline SF & Shape Factor & WCF & Wavelet Center Frequency \\
KU & Kurtosis & MW & Morlet Wavelet \\
RMS & Root Mean Square & BPFO & Bearing Pass Frequency Outer \\
CF & Crest Factor & BPFI & Bearing Pass Frequency Inner \\
SMF & Shape Modified Form & RF & Raw Form \\
ESPRIT & Estimation of Signal Parameters via & SORDINA & SOurce fRequency Detection via \\
& Rotational Invariance Techniques & & INvariance Approach \\
ENKU & ENvelope form through KUrtogram & IF & Instantaneous Frequency \\
IFESIS & $\begin{array}{l}\text { Instantaneous Frequencies Estimation } \\
\text { via Subspace Invariance properties of }\end{array}$ & & Fourier Transform \\
& wavelet Structures & & \\
\hline
\end{tabular}

\section{Introduction}

Bearing diagnostics has been a topic of intensive research for many years, presenting development and improvement of novel signal processing tools and algorithms for failure (anomaly) detection, classification and prediction, aiming at early and accurate fault detection in rotating machinery. The methods can be included in a framework of predictive maintenance focusing towards the increase of useful components life and the prevention of economic loss and catastrophic accidents. Feature extraction is one of the most basic steps in the fault diagnosis procedure. The signals emitted by rotating machinery are usually non-stationary, non-linear and with strong noise interference while the early signal energy is too low to extract fault features in time domain. In order to extract optimal features in low signal-to-noise ratio (SNR) environments, it is an urgent demand to develop effective and versatile signal processing tools which can be adapted to different applications and operating conditions. The widespread applications of rolling element bearings have inspired the emerging of many advanced technologies to monitor their health status. As a consequence, the last decades have seen the rapid advancement of feature extraction methods [1 - 8], such as time-domain methods, frequency domain methods and time-frequency methods, which are capable of extracting the most representative features and simultaneously decreasing their dimension in order to maximize the effectiveness and the accuracy of the classification methods.

In this work, the singular value is the extracted feature, used for novelty detection. This feature has not been widely used in the area of the engineering [9 - 12]. Kang et al. [9] introduced a robust feature extraction method to achieve high classification even in a noisy environment. The proposed method combines the Short-Time Energy with the Singular Value Decomposition (SVD) technique producing simple features used in a wide variety of classification schemes but the irregular patterns of their values can lead to low classification accuracy. For this reason, the SVD-based feature extraction approach is applied to overcome this limitation. The original signal is divided into sub-bands, the feature values are calculated in each sub-band and a matrix is composed. Afterwards, this matrix is decomposed using SVD. The Singular Values (SVs) are used as features to categorize the faults.

A new fault feature approach is introduced in [10]. This work is based on the Singular Spectrum Analysis of the vibration signal that is implemented in order to decompose the acquired signals into an additive set of principal components. Initially, the vibration signal is mapped into a sequence of multidimensional lagged vectors resulting to a trajectory matrix. This matrix is decomposed through SVD into a sum of mutually orthogonal, unit rank, elementary matrices. The obtained SVs, set in decreasing order, are used to form a Singular Spectrum. Then, the Singular Spectrum plots for different bearing conditions are compared and the appropriate SVs are selected. Finally, they are adopted as inputs to an artificial neural 
network for fault diagnosis.

However, most of the available datasets are captured during normal operating conditions. Additionally, an inevitable consequence of the high degree of system complexity is the large number of possible failure modes, the effects of which on observable data are often poorly defined. Thus, there are insufficient examples of failure to construct accurate fault-detection systems. As a result, conventional fault-specific failure-detection schemes are usually limited to identifying a small subset of known, well-understood modes of failure. An alternative to identifying rare and unexpected modes of failure is the novelty detection approaches [13 - 15], where a model of normality is constructed from normal system data. Departures from abnormal behavior are classified as novel events. Novelty detection is alternatively known as one-class classification, outlier detection or anomaly detection. Being novelty detection methods able to work when only normal data are available, such methods are of considerable promise for health monitoring in the case of lacking fault samples and prior knowledge.

A number of classifiers (Gaussian Mixture Models, Hidden Markov Models, kNN-based, etc) have been adapted for novelty detection [16 - 19]. It has been realized in practice that the novelty detection is an extremely challenging task. As a result several novelty detection models have been proposed performing well on specific data sets. It is clearly evident that till now there is no global best model for novelty detection and the success depends not only on the type of the method used but also on the statistical properties of data handled.

In this study, conventional control charts are applied and configured for successful anomaly detection. In contrast to condition monitoring and fault diagnosis of rotating machinery, monitoring of chemical processes using conventional control charts has been reported in literature by many researchers [20]. In industrial processes, conventional control charts such as Shewhart control chart are well established for the monitoring of univariate processes. In order to monitor multivariable processes, multivariate statistical process control (MSPC) has been developed such as the Hotelling $\mathrm{T}^{2}$ control chart for correlated variables.

A recent work about fault detection in mechanical engineering systems using control charts has been presented by Zhou et al. [21]. The researchers developed a damage detection scheme by integrating the recurrence plot method and $\mathrm{T}^{2}$ control chart. Five types of features are extracted by the recurrence quantification analysis to quantify the vibration signal characteristics. Then, a novel $\mathrm{T}^{2}$ bootstrap control chart is applied to monitor these features.

In this paper, a novel type of diagnostic features is proposed combining the morphological analysis and the Complex Shifted Morlet Wavelets. The extracted singular values are adopted as the optimal features. Then, the features are further used as inputs to univariate control charts focusing towards an early abnormal change detection and classification, under the assumption that measurements under normal machine operating conditions are available. The rest of the paper is organized as follows: the basic theory of the morphological analysis, the Complex Shifted Morlet Wavelets, the IFESIS and the SORDINA are summarized in Section 2. The complete hybrid methodology is presented in Section 3. The effectiveness of the proposed hybrid method is validated in Section 4, using two different experimental cases with bearing faults that are available online at the Prognostics Center of Excellence [22]. Finally the conclusions of this work are briefly mentioned in Section 5 . 


\section{Basic theory}

\subsection{Morphological Analysis}

\subsubsection{A brief review of Morphological Operators}

Mathematical Morphology (MM) [23] is a nonlinear spatial analysis method that analyzes the shapes of objects. According to the theory of MM, two factors are determinative for morphological analysis: the Morphological Operator (MO) and the Structuring Element (SE). Thus, the fundamental concept of the morphological signal processing is the modification of the shape of the signal through its interaction with the SE. The basic operators of MM include dilation, erosion, opening and closing [23, 24]. These MOs are related to Minkowski set operations and are used to construct morphological filters.

Using the definitions of Minkowski addition and subtraction between functions, the four basic morphological operations are defined as:

$$
\begin{aligned}
& \operatorname{dil}(f, g)=\left(f \oplus g^{r}\right)(x)=f(x) \oplus g(-x)=\sup _{y \in D}\{f(y)+g(y-x)\} \\
& \operatorname{er}(f, g)=\left(f \ominus g^{r}\right)(x)=f(x) \ominus g(-x)=\inf _{y \in D}\{f(y)-g(y-x)\} \\
& \operatorname{cl}(f, g)=(f \bullet g)(x)=\left[\left(f \oplus g^{r}\right) \ominus g\right](x) \\
& o p(f, g)=(f \circ g)(x)=\left[\left(f \ominus g^{r}\right) \oplus g\right](x)
\end{aligned}
$$

where $g^{r}(x)$ denotes the reflected (symmetric) function $g(x)$ with respect to the origin of the $\mathrm{x}$-axis. Equations (1) - (4) can be significantly simplified, if $g(x)$ is an even function, i.e. $g(x)=g(-x)$, and if sampled functions are used. For a signal $f(k)$, defined over a domain $D f$, and for a function $g(u)$ (called the structuring element) of length $L$ over a domain $D g$, the dilation and the erosion of the signal $f(k)$ by the element $g(u)$ are defined as:

$$
\begin{aligned}
& \operatorname{dil}(k)=(f \oplus g)(k)=\max _{u \in D g}\{f(k+u)+g(u)\} \\
& \operatorname{er}(k)=(f \ominus g)(k)=\min _{u \in D g}\{f(k+u)-g(u)\}
\end{aligned}
$$

Similarly, based on erosion and dilation, the closing and the opening operations of the signal $f(k)$ by the element $g(u)$ are further defined as:

$$
\begin{aligned}
& c l(k)=(f \bullet g)(k)=\operatorname{er}(\operatorname{dil}(k)) \\
& o p(k)=(f \circ g)(k)=\operatorname{dil}(\operatorname{er}(k))
\end{aligned}
$$

In practice, morphological operators are chosen based on different application scenarios of signal processing. Sometimes it is difficult to obtain the prior knowledge of the positive or negative impulsive features from a signal, especially when it has both positive and negative impulsive features. In this case, new combinations of the four operators are needed to be defined, such as the average filter.

According to Zhang and Yang [25], the shapes of the SE have little effect on the analysis, so in order to simplify the computation, the well established flat SE [24,26] is applied in the proposed methodology. According to studies on the MA [24 - 27] the length of the SE is an important key parameter. Considering 
a short length, much more impulse features can be extracted by the signal, while much noise may be retained. In the case of significant low SNR, the demodulation of a signal using a flat SE may be difficult and some useful information may be inhibited. Based on the aforementioned remarks, a flat SE can be used for industrial applications where the measured responses of machinery with faulty parts are corrupted by low to medium noise .

\subsubsection{Morphological gradient of Beucher}

The Morphological Operators are chosen in accordance with the application scenarios of the signal processing. In the case of defective bearings, the Beucher Gradient (BG) is a suitable MO to detect peaks [28]. Beucher gradient yields the maximum variation of the gray level intensities within the neighborhood defined by the SE rather than the local slope. This morphological gradient is defined as the arithmetic difference between the dilation and the erosion operators by the SE.

$p(k)=\operatorname{dil}(k)-\operatorname{er}(k)$

The shape, the length (domain) and the height (amplitude) of SE should be selected according to the analyzed signal. Morphological operations using flat structuring elements seem to be quite appropriate for the detection of peaks of impulsive type vibration signals, resulting by faulty bearings [24]. Thus, in this work, a flat SE is used [24]. Using a flat SE, the only parameter to be selected is the length $l$ that is very crucial. Considering much shorter length, much more impulse features will be extracted from the signal while much more noise will be retained. For the selection of the SE's length, the Kurtosis Criterion (KUC), introduced by Raj et al. [28] is used. The signal is morphologically filtered with a series of flat SEs of different lengths ranging from 0.1 to 0.9 of the minimum pulse period from either the Inner Race (IR) or the Outer Race (OR) fault. The morphologically filtered signal with the maximum KU value is selected for further analysis. The maximum KUC value indicates the signal with the highest number of peaks and the more useful fault information in its structure. Another method to determine the optimal parameter of the flat SE has been proposed in [29].

\subsection{Theoretical background of Complex Shifted Morlet Wavelets}

The complex Morlet wavelet can be defined in the time domain as a harmonic wave with a frequency $f_{o}$ multiplied by a Gaussian time domain window [30]:

$\psi(t)=\frac{2 \sigma}{\sqrt{\pi}} e^{-\sigma^{2} t^{2}} e^{j 2 \pi f_{o} t}$

The Fourier Transform (FT) of a scaled Morlet wavelet is purely real and is given by:

$\Psi(\alpha f)=2 e^{-\frac{\pi^{2}}{\sigma^{2}}\left(\alpha f-f_{o}\right)^{2}}=2 e^{-\frac{\pi^{2}}{(\sigma / \alpha)^{2}}\left(f-\frac{f_{o}}{\alpha}\right)^{2}}$

The parameter $\sigma$ (standard deviation) controls the shape of the wavelet in both time and frequency domain. The adaptive wavelet resolution is evident in the fact that the center frequency and the bandwidth $\sigma$ both depend on the scale $\alpha$ (Eq. 11). The effective mother wavelet durations for a Morlet wavelet of Eq. 10 are $\Delta t_{\psi}=1 / 2 \sigma$ and $\Delta \omega_{\psi}=\sigma$ [31]. Having a Gaussian shape, the Morlet wavelet provides the 
optimal Heisenberg uncertainty box [31]: $\Delta t_{\psi} \Delta \omega_{\psi}=1 / 2$, and thus also the optimal time-frequency localization.

Eq. 11 demonstrates that the standard wavelet scaling approach leads to a relation between the MW center frequency $\left(f_{0} / \alpha\right)$ and its bandwidth $(\sigma / \alpha)$, through the scaling parameter $\alpha$. In [32] Nikolaou and Antoniadis suggested the use of a Complex Shifted Morlet Wavelet (CSMW); a modification of the standard Morlet wavelet, in which two new independent parameters are defined, namely $f_{c}$ and $f_{b}$, replacing $f_{0} / \alpha$ and $\sigma / \alpha$, respectively. Thus, the wavelet is shifted but not scaled and its FT is:

$\Psi(f)=2 e^{-\frac{\pi^{2}}{f_{b}^{2}}\left(f-f_{c}\right)^{2}}$

In this way, the $f_{c}$ can be varied while $f_{b}$ is kept constant, making the CSMW to act as a proper frequency domain filter with a bandwidth $f_{b}$. The effective time duration of the wavelet is still $\Delta t_{\psi}=1 / 2 f_{b}$.

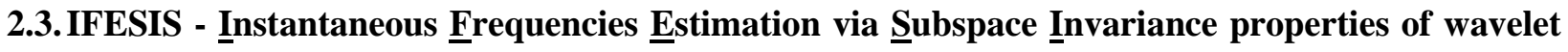 Structures}

Antoniadis et al. [30] proposed the IFESIS, a method for multi-component instantaneous frequency (IF) estimation, which combines the advantages of the CSMW with the advantages of the subspace based approaches. According to [30], a set of wavelet transforms of the signal is firstly obtained, using a structure of CSMW. In principle, the only constraint on the central frequencies and the bandwidths of the individual wavelets is that the number of the wavelets should be greater or equal to the number of the anticipated harmonic components. A number of complex signals equal to the number of the selected Morlet wavelets are extracted in the time domain. Each signal contains only the part of the signal frequency range which is allowed by the corresponding wavelet.

Initially, for the case of an impulsive signal, the WT of the signal is obtained using a CSMW. This leads to a complex signal $w(t)$ :

$w(t)=\int_{-\infty}^{+\infty} y(\tau) \psi(t-\tau) d \tau$

where

$y(\tau)=\sum_{k=1}^{P} A_{k} e^{-\zeta_{k} \omega_{n k} \tau} \cos \left(\omega_{d k} \tau+\phi_{k}\right)$

and

$\psi(t-\tau)=\frac{2 f_{b}}{\sqrt{\pi}} e^{-f_{b}^{2}(t-\tau)^{2}} e^{j 2 \pi f_{c}(t-\tau)}$

$P$ is the number of the excited frequencies $\omega_{n k}$. The damped frequency $\omega_{d k}$ is equal to $\omega_{n k} \sqrt{1-\zeta_{k}^{2}}$, $\omega_{c}=2 \pi f_{c}, \zeta_{k}$ denotes the damping ratio, $A_{k}$ and $\phi_{k}$ are respectively the amplitude and the phase shift of the $k$ resonance frequency. The convolution integral of Eq. 13 boils down to a Gaussian integral and can be solved analytically [33], yielding: 
$w(t)=\sum_{k=1}^{P} \frac{A_{k}}{2} g_{k} e^{-\zeta_{k} \omega_{n k} t} e^{j\left(\omega_{d k} t+\phi_{k}\right)}$

with

$g_{k}=e^{-\left(\frac{\omega_{c}-\omega_{n k}}{2 f_{b}}\right)^{2}} e^{-\frac{\omega_{c} \omega_{k}}{2 f_{b}^{2}}\left(1-\sqrt{1-\zeta_{k}^{2}}\right)} e^{j \beta_{k}}$

where

$\beta_{k}=\frac{\left(\omega_{c}-\omega_{n k}\right) \zeta_{k} \omega_{n k}}{2 f_{b}^{2}}$

The obtained expression for $w(t)$ is based on a single wavelet with parameters $f_{c}$ and $f_{b}$. The number of the wavelets, used for the analysis of the signal, is denoted by the parameter $M$. Each wavelet is characterized by its own parameters $f_{c i}$ and $f_{b i}$. Consequently, in case $M$ is more than 1 , a wavelet transformed complex signal $w_{i}(t)$ (where the index $i$ runs from 1 to $M$ ) should be calculated for each wavelet. Hence, Eqs. 16, 17 and 18 become:

$w_{i}(t)=\sum_{k=1}^{P} \frac{A_{k}}{2} g_{i k} e^{-\zeta_{k} \omega_{n k} t} e^{j\left(\omega_{d k} t+\phi_{k}\right)}$

with

$g_{i k}=e^{-\left(\frac{\omega_{c i}-\omega_{n k}}{2 f_{b i}}\right)^{2}} e^{-\frac{\omega_{c i} \omega_{k}}{2 f_{b i}^{2}}\left(1-\sqrt{1-\zeta_{k}^{2}}\right)} e^{j \beta i_{k}}$

where

$\beta_{i k}=\frac{\left(\omega_{c i}-\omega_{n k}\right) \zeta_{k} \omega_{n k}}{2 f_{b i}^{2}}$

The set of wavelet transforms, resulting from a cluster of $M$ CSMWs, can be unified in a matrix $\mathbf{W}$ of size $M \times L$, where $L$ is the number of samples in $w_{i}(t)$ :

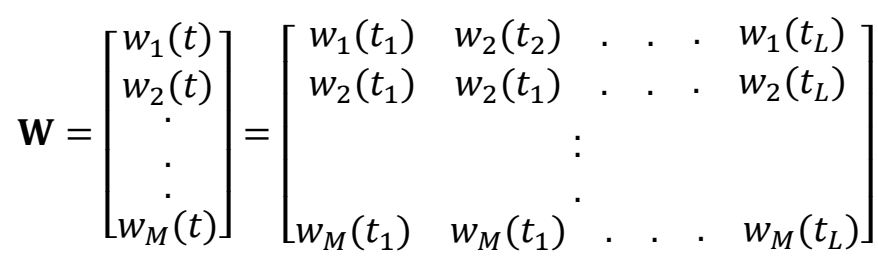

$R$ is another important parameter of the proposed procedure. In case $R$ is greater than one, each CSMW acts on each of the excited frequencies in the fashion described above. The result is a block matrix $\mathbf{W}$ with dimensions $M \cdot R \times L$, where all $R$ individual WT matrices of size $M \times L$ (as given in Eq. 22) are stacked together. 
$\mathbf{W}=\left[\begin{array}{c}\mathbf{W}^{1} \\ \mathbf{W}^{2} \\ \vdots \\ \mathbf{W}^{\mathrm{R}}\end{array}\right]$

As a next step, uniform sampling time $\Delta t$ is assumed, such as the n-th time instant is given by: $t_{n}=$ $n \Delta t$. Going back to Eq. $19, w_{i}\left(t_{n}\right)$ can be expressed as:

$w_{i}(n \Delta t)=\sum_{k=1}^{P} g_{i k} s_{k}$ and $i=1,2, \ldots, M$

where

$s_{k}=\frac{A_{k}}{2} e^{j \phi_{k}} q_{k}^{n}$

with

$q_{k}=e^{j \omega_{n k} T}$

$g_{k}=e^{-\left(\zeta_{k} \omega_{n k} \Delta t+j \omega_{d k} \Delta t\right)}=e^{\left(-\zeta_{k} \omega_{n k} \Delta t+j \omega_{n k} \sqrt{1-\zeta_{k}^{2}} \Delta t\right)}$

Eq. 27 leads to a crucial observation. The instantaneous excited frequencies $\omega_{n k}$ and the damping ratios $\zeta_{k}$ can be obtained from $q_{k}$ using the formulas:

$\omega_{n k}=\frac{\left|\ln \left(q_{k}\right)\right|}{\Delta t}$

$\zeta_{k}=\frac{\operatorname{Re}\left\{\ln \left(\mathrm{q}_{\mathrm{k}}\right)\right\}}{\omega_{n k} \Delta t}$

Furthermore, the n-th column of $\mathbf{W}$ (as defined in Eqs. 22 and 23) contains the complete set of wavelet transformed signals at time step $t_{n}=n \Delta t$. Based on Eq. 24, the following matrix equation can be introduced:

$\vec{W}\left(t_{n}\right)=\mathbf{G} \cdot \vec{s}$

where the two-index elements $g_{i k}$ are stored in the matrix $\mathbf{G}$, whereas $\vec{s}$ contains the one-index elements $s_{k}$. Now, considering the next time step $t_{n+1}=(n+1) \Delta t$, it follows from Eqs. 25 and 29 that:

$\vec{W}\left(t_{n+1}\right)=\mathbf{G} \cdot \boldsymbol{\Phi} \cdot \vec{s}$

with 
$\boldsymbol{\Phi}=\left[\begin{array}{llll}q_{1} & & & \\ & q_{2} & & \\ & & \ddots & \\ & & & q_{k}\end{array}\right]$

The equation (32) shows that the diagonal matrix $\boldsymbol{\Phi}$ can be regarded as a rotation operator, as it connects the two temporally displaced vectors $\vec{W}\left(t_{n}\right)$ and $\vec{W}\left(t_{n+1}\right)$ [34]. Knowledge of the diagonal values (also eigenvalues) of $\boldsymbol{\Phi}$ is sufficient for obtaining the instantaneous frequencies and amplitudes at each time step through Eqs. 28 and 29. Therefore, based on the rotational invariance of the signal subspace (Eqs. 30 and 31), Antoniadis et al. [30] used the well-established ESPRIT algorithm [34], in order to isolate the signal subspace from the noise subspace and to obtain the diagonal values of $\boldsymbol{\Phi}$. The procedure consists of the following steps:

1. A data matrix $\mathbf{Z}$ is formed at each time step:

$\mathbf{Z}\left(t_{n}\right)=\left[\begin{array}{lllll}\vec{W}\left(t_{n-N+1}\right) & \vec{W}\left(t_{n-N+2}\right) & \cdot & \cdot & \cdot \vec{W}\left(t_{n-1}\right) \\ \vec{W}\left(t_{n-N+2}\right) & \vec{W}\left(t_{n-N+3}\right) & . & \cdot & \cdot \vec{W}\left(t_{n}\right)\end{array}\right]$

A subset of the WT matrix $\mathbf{W}$ with length $N$ fills the top half of $\mathbf{Z}$. Another subset with the same length displaced forward by one time sample forms the bottom half of the data matrix. $N$ is a parameter which is manually selected by the user and defines the number of samples used to construct the Morlet wavelet.

2. A singular value decomposition (SVD) of $\mathbf{Z}$ is performed:

$\mathbf{Z}=\boldsymbol{U} \boldsymbol{D} \boldsymbol{V}^{T}$

where the matrices dimensions are respectively $\mathbf{U}: 2 M R \times 2 M R, \mathbf{D}: 2 M R \times N$ and $\mathbf{V}^{\mathrm{T}}: N \times N$. The diagonal elements of $\mathbf{D}$ are termed singular values (SVs). The number of the non-zero SVs is equal to the amount of the excited frequencies while $\mathbf{U}$ and $\mathbf{V}$ contain the left- and right-singular vectors of $\mathbf{Z}$.

3. $P$ denotes the number of signal's excited components to be identified taking values between 1 and the total number of excited frequencies due to a machinery fault. Moreover, since $\mathbf{D}$ has $N$ columns, $N>P$ should be satisfied. Once $P$ is fixed, the eigenvectors of $\mathbf{U}$ corresponding to the $P$ largest singular values are singled out and arranged in a matrix:

$\boldsymbol{U}_{\boldsymbol{p}}=\left[\begin{array}{llllll}\overrightarrow{u_{1}} & \overrightarrow{u_{2}} & \cdot & \cdot & \cdot \overrightarrow{u_{P}}\end{array}\right]$

The eigenvectors contained in $\boldsymbol{U}_{\boldsymbol{p}}$ span the signal subspace, while the remaining $(N-P)$ eigenvectors of $\mathbf{U}$ span the noise subspace and are therefore not included.

4. Next, $\boldsymbol{U}_{\boldsymbol{p}}$ is partitioned:

$U_{p}=\left[\begin{array}{l}U_{1} \\ U_{2}\end{array}\right]$ 
with dimensions $\boldsymbol{U}_{\mathbf{1}}: M R \times P$ and $\boldsymbol{U}_{\mathbf{2}}: M R \times P$.

5. In a next step, a matrix $\boldsymbol{\Psi}$ and $\boldsymbol{\Phi}$ are obtained:

$\boldsymbol{\Psi}=\left(\boldsymbol{U}_{\mathbf{1}}^{T} \boldsymbol{U}_{\mathbf{1}}\right)^{-1} \boldsymbol{U}_{\mathbf{1}}^{T} \boldsymbol{U}_{\mathbf{2}}$

The rotational invariance properties of $\boldsymbol{\Psi}$ and $\boldsymbol{\Phi}$ dictate that they have the same eigenvalues [34].

6. Finally, the eigendecomposition of $\boldsymbol{\Psi}$ is performed. Based on the rotational invariance properties of $\boldsymbol{\Phi}$ and $\boldsymbol{\Psi}$, the eigenvalues of $\boldsymbol{\Psi}$ are the same as those of $\boldsymbol{\Phi}$ [34]. Hence, the instantaneous excited frequencies $\omega_{n k}$ can be recovered from the eigenvalues $q_{k}$ of $\boldsymbol{\Phi}$.

Contrary to the classical subspace methods which are applied to the signal itself, this method proposes the application of the algorithm on the signal after its transformation by an appropriate wavelet structure. In this way, the performance of the subspace based approach is increased, since the desired time-frequency features of the signal are enhanced, while simultaneously, the undesired frequency components, as well as the noise, are suppressed. The method provides a means for the calculation of the number of resulting harmonic components. The number of the nonzero SVs of the corresponding SVD problem, which results as a consequence of the signal subspace invariance property, can be used as such an indicator. Moreover, since the IF calculations are performed at each time instant, the resulting Singular Values are also time dependent, providing valuable information on the possibly time variable dynamic structure of the signal.

\subsection{SORDINA - SOurce fRequency Detection via INvariance Approach}

A machine with a defective bearing exhibits vibratory responses with characteristic fault frequencies that can be calculated by formulas that assume no slippage of the rollers. However, in real world applications the actual characteristic fault frequencies differ from the nominal ones because of the slippage. Moreover, according to [35], it is important the center frequency (FC) of the Morlet window to be close to the harmonic frequency. Hence, the average value of the produced IF will be close to the peak frequency of the actual harmonic component.

In order to address these problems, a technique called SORDINA is introduced in order to detect the actual source frequency and its harmonics. The SORDINA is a CSMW-based technique and an extension of the IFESIS [30]. The basic concept of this approach is to find the actual fundamental fault frequency and its harmonics into selected frequency bands $\Delta F_{i}=\left[F_{L i} \ldots F_{H i}\right]\left(i\right.$ represents the $i^{\text {th }}$ harmonic $)$ that enclose the nominal fault frequency or the harmonics of a calculated fundamental fault frequency.

In practice, this approach involves the shift of a Morlet window into a selected frequency band $\Delta F_{i}$ with a center frequency $f_{c o, i}(j)=F_{L i}(j)+d F_{i}(j-1)$. The $j$ ranges from 1 to $R$, where $R$ is the number of shifts of the Morlet wavelet into the $i^{\text {th }}$ frequency band. The $R$ is equal to or less than $\left[1+\frac{F_{H i}-F_{L i}}{d f}\right]$, where $d f$ is the frequency interval of the spectrum that is equal to the ratio of the sampling rate $f_{s}$ over the length $N$ of the measured signal. The initial wavelet center frequency (WCF) $f_{c o, i}(1)$ and the last WCF $f_{c o, i}(R)$ are respectively equal to $F_{L i}$ and $F_{H i}$ and the frequency shifting step is $d F$. The algorithm yields an array of none zero Singular Values (SVs) and an Instantaneous Frequency (IF) curve in each shift of the Morlet wavelet into the $i^{\text {th }}$ frequency band. A Mean Singular Value (MSV) is obtained by the averaged SVs of the produced SV array. The SV array and the IF curve are the products of the signal subspace invariance property derived by the CSMW based decomposition process. As a consequence, an 
array of MSVs will be constructed applying the CSMW in the whole $i^{\text {th }}$ frequency band $\Delta F_{i}$. At the same time, the variance of the estimated IF is calculated for each shifted MW.

The resulting MSVs depend on the signal's narrow frequency band which is restricted by the corresponding wavelet bandwidth. The MSV features capture the principal characteristics related to the inherent frequency structure of the machine fault pattern. Thus, the larger MSV represents the large amplitude components in the decomposition and the low-amplitude components of the signal are represented by the smaller MSV. Furthermore, a low variance of the produced IF indicates a possible harmonic component, while a higher variance corresponds to a random component or noise.

Thus, the simultaneous assessment of a high MSV and a low IF variance increases the possibility of a harmonic detection. As a consequence, the procedure calculates the difference of the normalized MSVs and the relative normalized IF variances. The higher positive difference is selected (a) as a possible source frequency and (b) as the FC of the applied MW in the IFESIS approach.

In addition, the process's accuracy is directly related to the frequency shifting step $d F$ and the bandwidth $f_{b, S}$ of the shifted Morlet window. According to [35], the MW should be narrow enough to exclude neighboring components. In that case, the above parameters can be defined as a function of the $d f$.

\subsection{A theoretical framework of continuous variable control charts}

Control charts [36] with respect to their design structure are classified into two categories: (a) memoryless and (b) memory control charts. The Shewhart-type charts, which ignore past information, are widely known and most often used in practice because of their simplicity and global performance. Cumulative sum (CUSUM) control charts and exponentially weighted moving average (EWMA) control charts are the two most commonly used memory control charts. Their control structure is set up such as they exploit the past information along with the current to give a better performance for small and moderate shifts.

\subsubsection{Shewhart control charts}

Shewhart-type charts are the most popular charts in practice because of their simplicity, ease of application, and the fact that these versatile charts are quite efficient in detecting moderate to large shifts. Shewhart control charts use the arithmetic mean as the centre line (CL). The mean is calculated by taking the sum of all values and dividing by the number of values. As the relative distances from the mean are taken into consideration, Shewhart charts are a sensitive way of detecting whether observed variation is due to common or special causes. These control charts also contain control limits. The control limits define the boundaries of expected common cause variation around the mean. If the data points are randomly arranged within the control limits, Shewhart charts allow for a kind of future performance prediction. The control limits are calculated statistically from the data referred as the lower control limit (LCL) and the upper control limit (UCL) on a control chart. Control limits define the zone where the observed data of a stable and consistent process occurs. Following the commonly used rule, by default the limits are set at 3-sigma.

Depending on the type of data being plotted: (a) attributes (counts) or (b) variable (continuous), and the purpose of the analysis, different types of Shewhart control charts can be used. The Average or X-bar chart is one of the most common control chart that monitors and displays the changes in the average output of a process/variable feature. 


\subsubsection{The classical EMWA control chart}

Another popular class of control charts is the EWMA charts [37]. The EWMA charts also take advantage of the sequentially accumulating nature of the data and are known to be efficient in detecting smaller shifts but are easier to implement compared to the CUSUM charts. While Shewhart charts consider only the most recent test data point to determine whether the statistical limits have been exceeded or not, EWMA charts consider all previous points using a weighing factor that makes the outcome more sensitive to the influence of recent points. The recursive formula for EWMA is:

$E W M A_{i}=c \cdot x_{i}+(1-c) \cdot E W M A_{i-1}$

where $c$ is a constant weighting factor and $x_{i}$ is the current variable value. In case the EWMA weighting factor is set equal to one, a Shewhart control chart is estimated.

\subsection{Spectra Averaging}

The vibration signals of a machine always carry the dynamic information of the machine. These signals are very useful for feature extraction and fault diagnosis. However, in many cases the very low SNR renders the extraction of feature components difficult and the applicability of information finally drops down. To reduce the noise effects and to improve the SNR, it's possible to average a number of successive spectra $S$. By averaging, the peaks corresponding to random events and additive noise are reduced, while the peaks corresponding to periodic phenomena are amplified and revealed. Since averaging a number of multiple spectra, stationarity over a longer period is assumed. The larger the number of FFTs being averaged, the closer the individual noise-power bin values will approach the true average noise power. A simple and powerful ensemble average of spectrum values of each frequency is used:

$S_{\text {aver }}=\frac{1}{N} \sum_{i=1}^{N} S_{i}$

where $\mathrm{N}$ is the number of the averages and $S_{i}$ the $\mathrm{i}^{\text {th }}$ spectra.

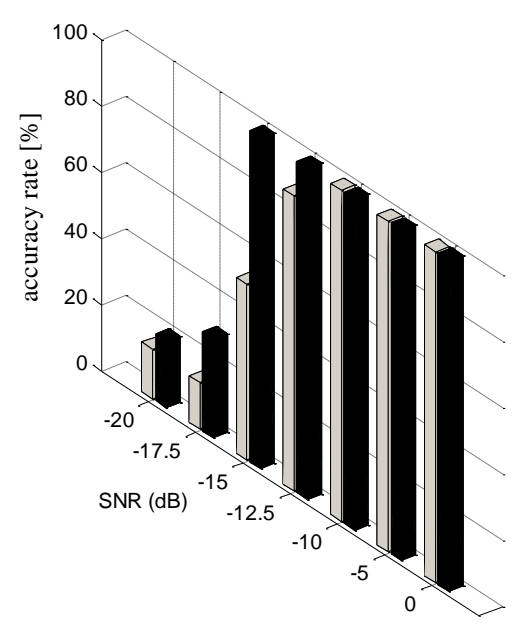

(a)

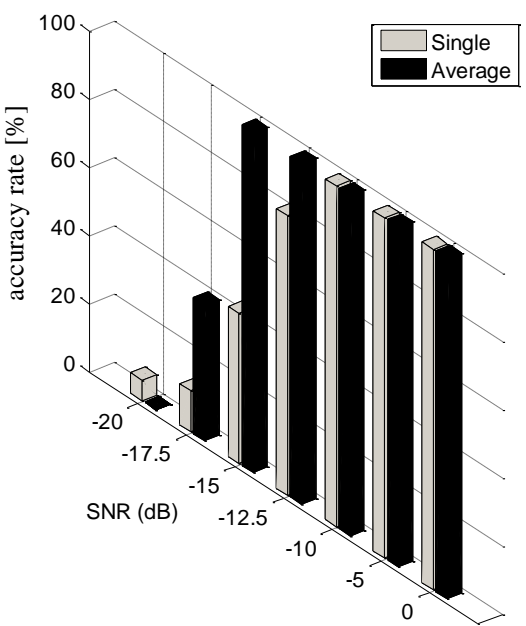

(b)

Fig. 1: Spectra averaging performance for frequencies (a) close to DC and (b) away from DC. 
The sensitivity of spectra averaging performance with respect to the SNR is further analysed by varying systematically the above parameter using the bearing simulation model in [38]. The dynamic response resulting from an induced defect in a bearing can be expressed by:

$d(t)=\left\{\begin{array}{l}\sum_{k=0}^{N} q(t) A_{k} \delta\left(t-k T_{d}-\tau_{k}\right) \otimes \sum_{i=1}^{M} B_{i} e^{-2 \pi \zeta_{i} f_{n} t} \cos \left(2 \pi f_{o i} t\right)+n(t), \text { for } I R \\ \sum_{k=0}^{N} A_{k} \delta\left(t-k T_{d}-\tau_{k}\right) \otimes \sum_{i=1}^{M} B_{i} e^{-2 \pi \zeta_{i} f_{n} t} \cos \left(2 \pi f_{o i} t\right)+n(t), \text { for } O R\end{array}\right.$

where $q(t)$ is the distribution of the load, $A_{k}$ is the amplitude of the impacts generated during the contact of the rolling elements with the defect, $\delta(t)$ is the dirac function, $T_{d}$ is the period of the fault, $\tau_{k}$ takes into account the slip of rolling element bearings, $B_{i}$ is the amplitude of the response of a 1DOF system to the impact excitation, $\zeta_{i}$ is the damping factor, $f_{n}$ is the natural frequency and $f_{o}$ is the damped natural frequency. Normalized sampling parameters are used:

The model equations are described in details in [38]. The different values of the fundamental fault frequency $f_{d}$ are selected a) close and b) away from the DC. A set of different levels of additive noise are considered, with SNR values ranging from - $20 \mathrm{~dB}$ up to $0 \mathrm{~dB}$. Initially, $M$ clusters, consisting of 10 simulated bearing responses with a specific SNR, are constructed. Then, the 10 contaminated simulations of each cluster are transformed by the morphologically filtering and their spectrums are averaged. Thus, $M$ single shape modified simulations are reconstructed. Afterwards, through the proposed hybrid approach, the maximum MSVs in the fundamental frequency band $\Delta F_{j, f_{d}, 1}$ of the $M$ averaged shape modified simulations and each one of their 10 relative raw simulations are detected. The aggregated results are presented in Fig. 1. The accuracy rate $(\%)$ of the proposed methodology is amplified by the implementation of the spectra averaging. Taking advantage of spectra averaging, the additive noise from the vibration signal and the useful source signals can be enhanced for fault diagnostics. Thus, important signal features can be obtained efficiently by the proposed hybrid approach using the spectra averaging. On the other hand, the effectiveness of the proposed method is reduced by the absence of the spectra averaging. Moreover, as shown in Fig. 1, the fault frequency value does not affect the performance of the hybrid feature extraction method.

\section{Overall architecture of the feature extraction and novelty detection method}

In this work, an abnormal change tracking and taxonomy process is introduced for bearing performance degradation assessment and classification. The proposed hybrid framework involves two primary modules: (1) the feature extraction, as presented in Fig. 2, and (2) the abnormal change detection and fault classification, as shown in Fig. 3. Additionally, the second primary module includes two main parts: (2.A) the offline modeling/training and (2.B) the online monitoring/testing and update.

\subsection{Feature extraction}

In this section, the proposed feature extraction scheme is briefly presented. The basic architecture is illustrated in Fig. 2. Initially, a set $i$ of $N$ sequential measured signals is transformed by morphologically filtering, through their interaction with a flat SE. The nominal characteristic fault frequencies $\mathrm{BPFO}^{\mathrm{TH}}$ and $\mathrm{BPFI}^{\mathrm{TH}}$, the rotational shaft frequency $\mathrm{RPM}^{\mathrm{TH}}$ and the sampling frequency $f_{s}$ are used as inputs to the algorithm of the morphological analysis. The length of the flat SE is estimated by applying the KUC [28] for the shorter impulse period between the IR and OR fault period. The success of this approach is due to 
the fact that the Beucher gradient cuts off the high-frequency resonances excited by the impacts and enhances the low-frequency components related to the mechanism of the defect. Hence, this approach offers a strong and reliable diagnostic potential as it concentrates the useful diagnostic information into a determinate low-frequency band (up to $1,000 \mathrm{~Hz}$ ).

Then, the shape modified signals are transformed using FFT. Using the spectra averaging, an effective denoising performance is achieved. Thus, a single shape modified signal with improved SNR is reconstructed through the IFFT of the averaged spectrum.

Afterwards, this new shape modified signal $j$ is inducted to SORDINA. Specifically, this step is used to detect the actual harmonics of the shaft frequency, the OR and the IR fault frequencies. First, a MW with a bandwidth $f_{b, S}$ equal to $(2 \div 3) d f$ shifts with a frequency shifting step $d F=d f$ into three predefined frequency $\triangle F_{j, R P M, 1}=\left[R P M^{T H} \pm 5 \mathrm{~Hz}\right], \Delta F_{j, B P F O, 1}=\left[B P F O^{T H} \pm 10 \mathrm{~Hz}\right]$ bands and $\triangle F_{j, B P F I, 1}=$ $\left[B P F I^{T H} \pm 10 \mathrm{~Hz}\right]$. The FCs of the shifted MWs, which give the highest positive differences of the normalized MSVs and the relative normalized IF variances for each $\Delta F_{j, R P M, 1}, \Delta F_{j, B P F O, 1}$ and $\Delta F_{j, B P F I, 1}$, are selected to be the FCs of the MWs that will be applied in the IFESIS approach. Moreover, the SORDINA considers that these FCs could be the possible failure frequencies RPM ${ }^{\mathrm{M}}, \mathrm{BPFO}^{\mathrm{M}}$ and $\mathrm{BPFI}^{\mathrm{M}}$. Next, another MW under similar assumptions as previous is introduced into the frequency $\triangle F_{j, R P M, 2}=$ $\left[2 \cdot R P M^{M} \pm 5 \mathrm{~Hz}\right] \quad, \quad \Delta F_{j, B P F O, k}=\left[k \cdot B P F O^{M} \pm 10 \mathrm{~Hz}\right], k=2, \ldots, k_{2} \quad$ bands $\quad$ and $\Delta F_{j, B P F I, k}=$ $\left[k \cdot B P F I^{M} \pm 10 \mathrm{~Hz}\right], k=2, \ldots, k_{3}$. The maximum numbers $k_{2}$ and $k_{3}$ of the frequency $\triangle F_{j, B P F O, k}$ bands and $\triangle F_{j, B P F I, k}$ are analogous to the number of the BPFO and the BPFI harmonics in the frequency band up to $1,000 \mathrm{~Hz}$ in accordance with the BG performance. Again, the FCs of the shifted MWs that correspond to the highest positive differences of the normalized MSVs and the relative normalized IF variances for each $\triangle F_{j, R P M, 2}, \Delta F_{j, B P F O, k}$ and $\Delta F_{j, B P F I, k}$ will be used as FCs of the MW cluster in the IFESIS. As a consequence, three groups consisting of the selected FCs are constructed: $G_{j, R P M}(1 \times 2)$, $G_{j, B P F O}\left(1 \times k_{2}\right)$ and $G_{j, B P F I}\left(1 \times k_{3}\right)$. In case, there are harmonic components in the testing frequency bands $\Delta F_{j}$, the selected FCs of the corresponding $G$ groups will have high possibility to coincide with one of the failure frequencies, if these FCs are integer multiplies and the peaks of their corresponding tested differences dominate in the frequency bands. Otherwise, they will be just identical with random components or noise.

Thus, a cluster of $\left(k_{2}+k_{3}+2\right)$ Morlet windows is applied in the IFESIS approach. The bandwidth $f_{b, I F}$ of the MW is considered equal to $(5 \div 10) d f$ in order to take into account the leakage energy. Since the diagonal matrix produced by the eigendecomposition process allocates the SVs in a descending order, the IFESIS is applied separately to each one of the previous three groups. The price paid for this computational cost is compensated by the separation of the fault information to OR and IR. Otherwise, it would be impossible to distinguish the parameters for different types of fault as the order of the MSVs does not correspond to the input order of Morlet window frequency centers. Hence, this process is time consuming but more effective.

The resulting nonzero MSVs of the SVD problem are the optimal features that correspond to the machine heath condition. These prominent features are relevant to the type of the bearing defect providing valuable diagnostic and class information. Additionally, it is easily demonstrated that the MSVs features have the same sensitivity and importance degree during the clustering operation. They can, also, bring the immediate effect of the fault mechanism and maximize the performance of the learning model. Thereby, as shown in Fig. 2, (a) the local MSVs corresponding to the fundamental frequencies and their harmonics, 
(b) the cumulative MSVs corresponding to OR $\left(S V_{j, O R}=\sum_{i=1}^{k_{2}} S V_{j, i B P F O}\right)$ and IR $\left(S V_{j, I R}=\sum_{i=1}^{k_{3}} S V_{j, i B P F I}+\sum_{i=1}^{2} S V_{j, i R P M}\right) \quad$ defects and $\quad$ (c) the overall MSV $\left(S V_{j, T}=\sum_{i=1}^{k_{2}} S V_{j, i B P F O}+\sum_{i=1}^{k_{3}} S V_{j, i B P F I}+\sum_{i=1}^{2} S V_{j, i R P M}\right)$ corresponding to the bearing health condition are calculated.

Generally speaking, the IFESIS approach can be skipped, if the researcher decides $f_{b, S}=f_{b, I F}$. In this case, the MSV feature will be directly extracted by the SORDINA.

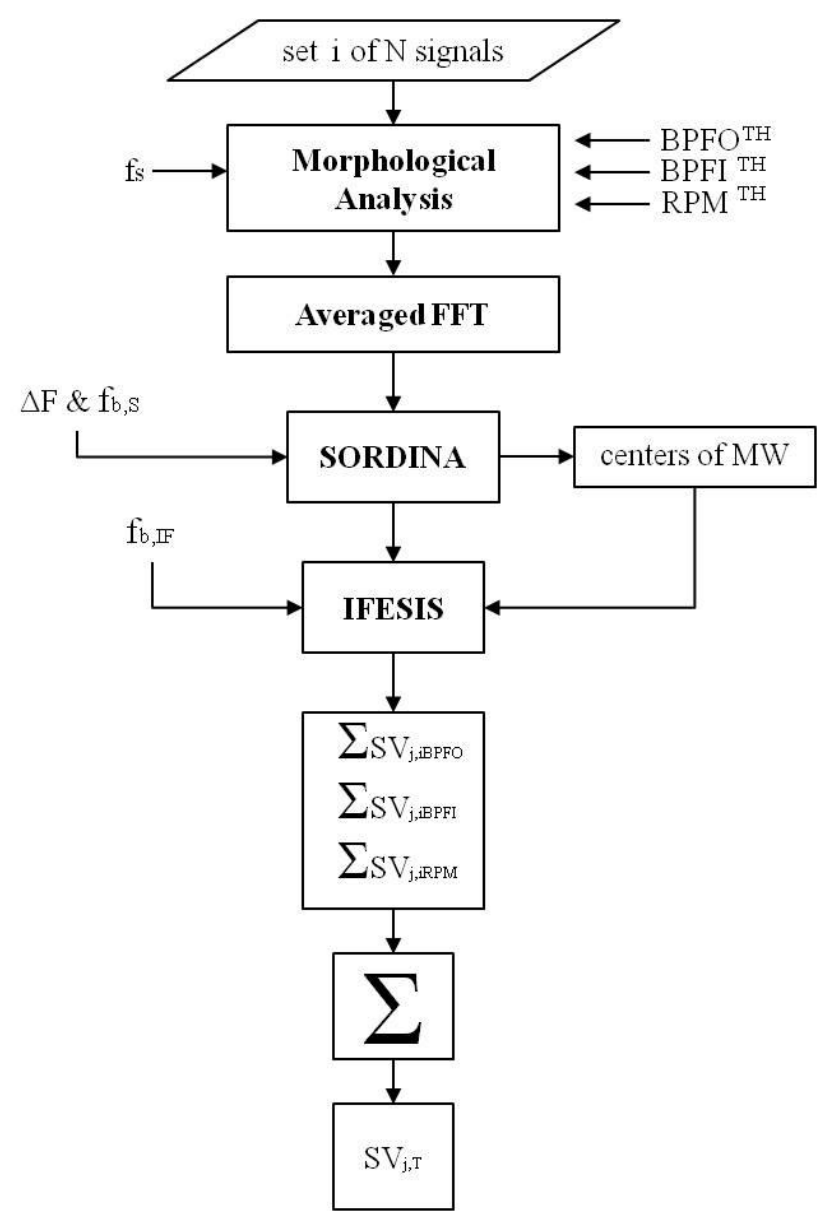

Fig. 2: Block diagram of the novel feature extraction process.

\subsection{Off-line modeling/Training}

During the offline training, the features $S V_{j, T}, j=1, \ldots, M$, obtained by the feature extraction approach for a series of $M$ normal sets $i$, are trained through the univariate EWMA or the X-bar control chart in order the estimated UCL to determine the threshold for the testing stage. The value of the UCL threshold can be updated each time the decision of the on-line monitoring for the new testing set $i$ is 'normal' (Fig. 3). 


\subsection{On-line monitoring/Testing}

At the on-line testing stage (Fig. 3), the novel feature extraction approach is employed to compute the key feature $S V_{j, T}$ for the set $i$ of the signals captured by a data acquisition system. The abnormal detector is triggered to test this overall MSV feature. A testing overall MSV feature is classified as normal and the machine is considered to be in a healthy operation condition if it is located below the estimated UCL threshold. Then, this overall MSV feature will be used to retrain the control chart model.

Hence, if no fault is detected, the 'fault classification part' of the testing module will not be activated. On the other hand, if the 'abnormal detection module' captures a testing overall MSV feature of a fault, it will activate the 'fault classification module' (Fig. 3). Once a fault is detected, a simple value comparison to $S V_{j, O R}$ and $S V_{j, I R}$ of the testing modified signal is applied. If $S V_{j, O R}$ is larger than $S V_{j, I R}$, the 'classification module' detects an OR fault, otherwise, the diagnosis results to an IR fault.

\section{Application to real world signals}

In order to evaluate the effectiveness of the proposed method for bearing fault diagnosis, two cases with different bearing fault types are considered in this study. The bearing data used for the analysis are available online at the Prognostics Center Excellence [22].

\subsection{Experimental data sets}

The sampling rate $f_{s}$ of the bearing data is $20 \mathrm{KHz}$ and the length $P$ is 20,480 samples. The rotation speed $\mathrm{RPM}$ is $2,000 \mathrm{rpm}$. The theoretical fault frequencies $\mathrm{BPFO}^{\mathrm{TH}}$ and $\mathrm{BPFI}^{\mathrm{TH}}$ are calculated equal to 236 and $297 \mathrm{~Hz}$, respectively. The signatures of the original set $T_{1}[22]$ acquired from the $5^{\text {th }}$ accelerometer mounted on the horizontal direction of the bearing No 3 and the vibratory data of the original set $T_{2}$ [22] coming from the $1^{\text {st }}$ sensor mounted on the horizontal direction of the bearing 1 are used for diagnosis. Figure 4 presents typical measured signals in the time domain demonstrating a medium SNR. An OR failure occurred on the bearing 3 of the set $T_{1}$ whereas an IR defect was detected on the bearing 1 of the set $T_{2}$. In both test cases, after detailed analysis, the time instance, when the defects started developing, is detected. Based on intensive analysis of the signals, the sequential measurements No 531 (Figs. 5(a) and 6(a)) and No 532 (Figs. 5(b) and 6(b)) of the data set $T_{1}$ experience the last healthy and the very first slight failure stage, respectively. As it can be observed in the spectrums of the normal (raw and modified) signal of $T_{1}$ (Figs. 5(a) and 6(a)), a peak close to $231 \mathrm{~Hz}$ corresponding to the possible actual $\mathrm{BPFO}^{\mathrm{M}}$ can be highlighted. Although bearing wear has not yet been developed, the fault frequency $\mathrm{BPFO}^{\mathrm{M}}$ is detected due to the 6,000 $\mathrm{lb}$ radial load placed on the shaft. This bearing overcharging is observed as early as the first measured signal of the set. Similarly, for the data set $T_{2}$, the measurement No 1,803 represents the last normal operating condition of the bearing and the next measurement No 1,804 delimits the beginning of the failure stage.

A sufficient number of signals are selected from the two original data sets. 61 signals from the set $T_{1}$ and

62 signals from the set $T_{2}$ are selected respectively constructing the new subsets $T_{1, n}$ and $T_{2, n}$. The signals are selected such as they cover relatively time-equidistantly the whole duration of the experiment from the beginning up to the complete failure of the bearing. The signals captured exactly before and exactly after the introduction of the damage are also included among the 61 and 62 selected signals. The signals No 45 and No 46 of the new subset $T_{1, n}$ correspond to the signals No 531 and No 532 of the original set $T_{1}$. Similarly, the signals No 40 and No 41 of the subset $T_{2, n}$ correspond to the signals No 1,803 and No 1,804 of the set $T_{2}$. 
The first 30 healthy measurements of each data set $T_{i, n}, i=1,2$ are used to construct a training data set $S_{t r}$ of 60 normal signals. The data of the $S_{t r}$ are used to train the corresponding control chart models, while the remaining 31 and 32 measurements of those data sets are used as the testing sets $S_{1}$ and $S_{2}$ for the on-line/testing stage. The first testing set $S_{1}$ includes 15 measurements corresponding to the normal operating condition and 16 measurements where slight and severity failures are present. Following the same approach, the second testing set $S_{2}$ consists of 10 normal and 22 faulty sequential measurements.

TESTING

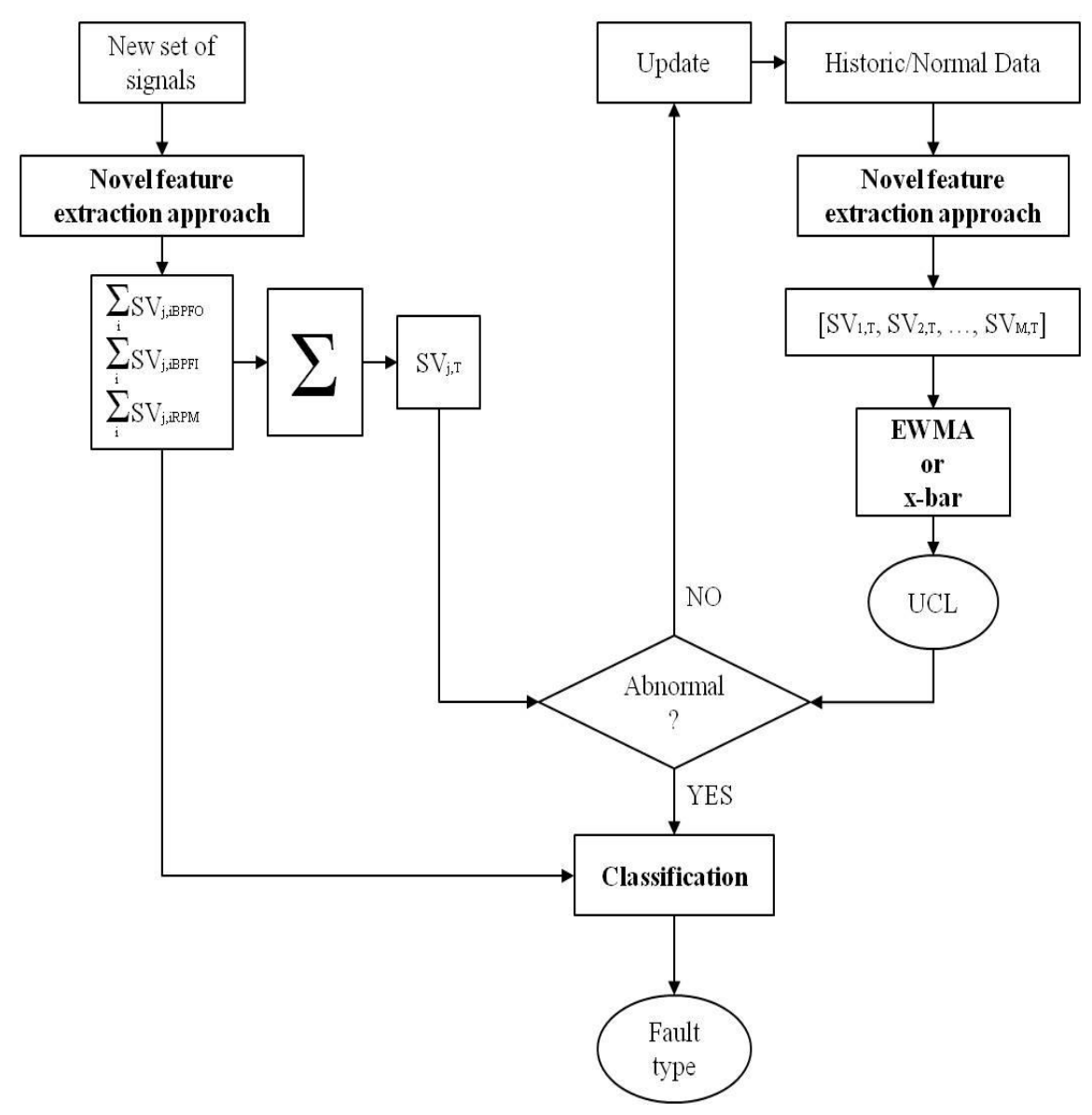

Fig. 3: Architecture of the proposed hybrid framework.

\subsection{Off-line/training process}

The FFT averaging process is skipped as it had not been envisaged for the data acquisition of the original data. Initially, the training data set $S_{t r}$ consisting of 60 normal measurements is preprocessed through the BG. Then, each shape modified signal $j(=1 \ldots 60)$ is introduced to SORDINA. A Morlet window with a bandwidth $f_{b, S}=2 d f=2\left(f_{s} / P\right)$ shifts with a frequency shifting step $d F=d f$ into the three predetermined frequency fields $\Delta F_{j, R P M, 1}, \Delta F_{j, B P F O, 1}$ and $\triangle F_{j, B P F I, 1}$. The three FCs of the shifted MWs corresponding to the highest positive proposed differences are selected to represent the possible actual 
$\mathrm{RPM}^{\mathrm{M}}, \mathrm{BPFO}^{\mathrm{M}}$ and $\mathrm{BPFI}^{\mathrm{M}}$ frequencies, respectively. Next another $\mathrm{MW}$ is introduced shifting into the frequency fields $\triangle F_{j, R P M, 2}, \Delta F_{j, B P F O, k}, k=2, \ldots, 4$ (= the number of BPFO harmonics in the frequency field up to $1,000 \mathrm{~Hz}$ ) and $\Delta F_{j, B P F I, k}, k=2,3$ (= the number of BPFI harmonics in the frequency field up to $1,000 \mathrm{~Hz}$ ). Again, the FCs of the MWs presenting the highest positive proposed differences for each $\triangle F_{j, R P M, 2}, \triangle F_{j, B P F O, k}$ and $\triangle F_{j, B P F I, k}$ are considered to be the harmonics of the $\mathrm{RPM}^{\mathrm{M}}, \mathrm{BPFO}^{\mathrm{M}}$ and $\mathrm{BPFI}^{\mathrm{M}}$ frequencies, respectively. Afterwards, for each normal modified signal $j$, the groups $G_{j, R P M}, G_{j, B P F O}$ and $G_{j, B P F I}$ are constructed consisting of 2, 4 and 3 FCs, respectively.

Thus, for each normal modified signal $j$, the corresponding FCs of the $3 G$ groups are used as MW frequency centers in the IFESIS approach, where the bandwidth $f_{b, I F}$ of the MWs is considered equal to $10 d f$. As a consequence, a cluster of $9 \mathrm{MW}$ is partially applied resulting to an overall MSV $S V_{j, T}$ corresponding to the healthy condition of a normal modified signal $j$. Finally, the total amount of the $S V_{j, T}, j=1, \ldots, 60$ obtained by this process are trained through the EWMA or the X-bar control chart in order to estimate the threshold UCL. The influence of the weight factor $c$ on the EWMA model is studied for a variety of values between 0 and 1. For illustration purposes of the EWMA results, the weighted factor $c$ of Eq. 38 is chosen equal to 0.6. The outcomes of the training process, $\mathrm{UCL}_{\mathrm{EWMA}}=1.164$ and $\mathrm{UCL}_{\mathrm{X} \text {-bar }}=1.265$ for $c=0.6$, are illustrated in Figs. 7 and 8, respectively.

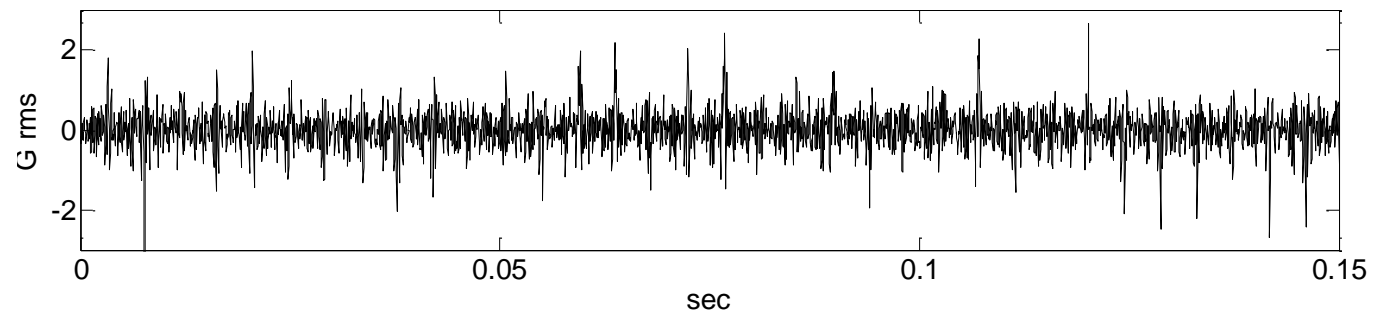

(a)

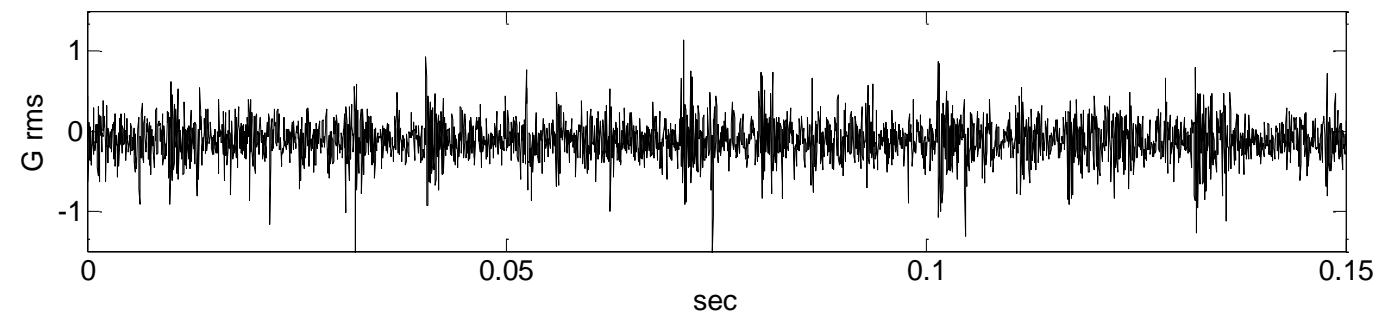

(b)

Fig. 4: Time-waveforms of the measured signals - (a) 2004.02.19.06.02 [22] of the $1^{\text {st }}$ data set and (b) 2004.11.25.16.07 [22] of the $2^{\text {nd }}$ data set.

\subsection{On-line/testing process and analysis of results}

First of all, it should be noted that each data set $S_{1}$ and $S_{2}$ is analysed separately through the part '2.B' (Fig. 3). The FFT averaging process is skipped as it had not been envisaged for the data acquisition of the original data.

Initially, each one of the 31 and 32 signals of the data sets $S_{1}$ and $S_{2}$, respectively, are transformed through the BG. Then, implementing the SORDINA approach to each modified signal $j$, the groups $G_{j, R P M}, G_{j, B P F O}$ and $G_{j, B P F I}$ are constructed consisting of 2, 4 and 3 FCs, respectively, selected on the 
basis of the proposed maximum differences. The calculated differences through SORDINA for three different testing signals of the data set $S_{1}$ are presented in the Figs. 9 - 14.

The SORDINA outcomes for the sample No 531 are shown in Figs. 9 and 10. Fig. 9 displays the computed MSVs, the variances of the estimated IFs and the differences of the normalized MSVs and the relative normalized IF variances for the frequency fields: (a) $\triangle F_{531, B P F I, 1}$, (b) $\Delta F_{531, B P F I, 2}$ and (c) $\triangle F_{531, B P F I, 3}$. The same outcomes are presented in Fig. 10 for the frequency fields: (a) $\triangle F_{531, B P F O, 1}$, (b) $\triangle F_{531, B P F O, 2}$, and (c) $\triangle F_{531, B P F O, 3}$.

As shown in Fig. 9(a), the highest difference for the frequency field $\triangle F_{531, B P F I, 1}$ is detected equal to $288.1 \mathrm{~Hz}$. This frequency diverges about $9 \mathrm{~Hz}$ from the $\mathrm{BPFI}^{\mathrm{TH}}$ and is selected as the possible fundamental fault frequency $\mathrm{BPFI}^{\mathrm{M}}$, while the lower differences are associated to random frequencies and noise. The $2^{\text {nd }}$ and $3^{\text {rd }}$ integer multiples of the selected frequency $\mathrm{BPFI}^{\mathrm{M}}=288.1 \mathrm{~Hz}$ are considered to be the centers of the frequency fields: (a) $\triangle F_{531, B P F I, 2}$ and (b) $\triangle F_{531, B P F I, 3}$, respectively. Then, the SORDINA is applied to these frequency fields resulting respectively in maximum differences in the frequencies $585 \mathrm{~Hz}$ and $862.3 \mathrm{~Hz}$, as shown in Figs. 9(b) and 9(c). These frequencies seem to be the $2^{\text {nd }}$ and the $3^{\text {rd }}$ harmonics of the $\mathrm{BPFI}^{\mathrm{M}}$. As it can be observed in Fig. 9 there is no obvious harmonic relationship among the selected frequencies. Moreover, it should be emphasized that the peaks of the maximum differences do not dominate in the $\Delta F$ frequency fields. These observations could be a semantic indication that the selected frequencies correspond to random components or noise. Additionally, these three frequencies are the FCs of the group $G_{531, B P F I}$.

Similar conclusions are drawn implementing the SORDINA in the frequency fields: (a) $\triangle F_{531, B P F O, 1}$, (b) $\triangle F_{531, B P F O, 2}$ and (c) $\triangle F_{531, B P F O, 3}$. At Fig. 10 similar patterns to those of Fig. 9 can be identified. As it can be observed in Fig. 10(a), the selected frequency $230.5 \mathrm{~Hz}$ is identical to the actual $\mathrm{BPFO}^{\mathrm{M}}$ due to the bearing overcharging under the $6,000 \mathrm{lb}$ radial load and not to the bearing wear. This explanation could also be supported by the 'non harmonic relationship' of the selected frequencies $230.5 \mathrm{~Hz}, 463.9 \mathrm{~Hz}$ and $683.6 \mathrm{~Hz}$ (Fig. 10) that constitute the FCs of the group $G_{531, B P F O}$.

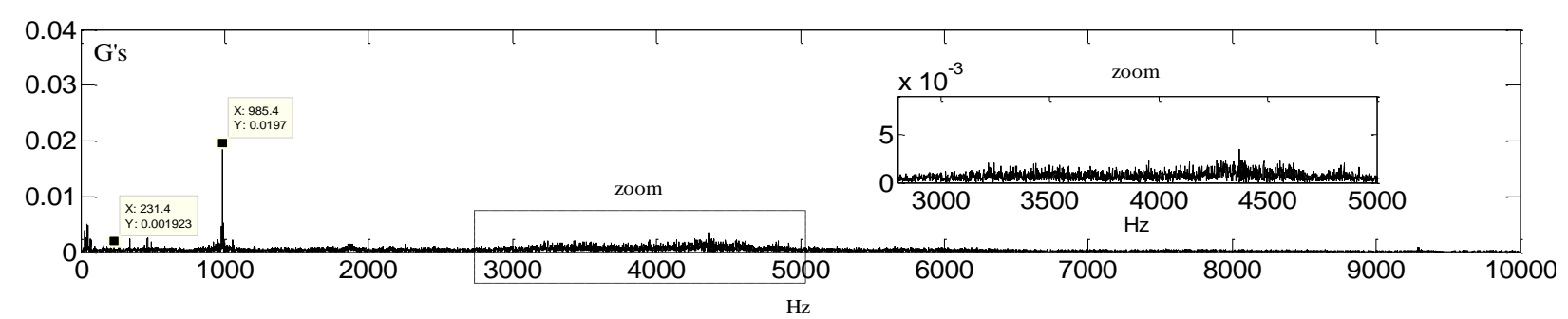

(a)

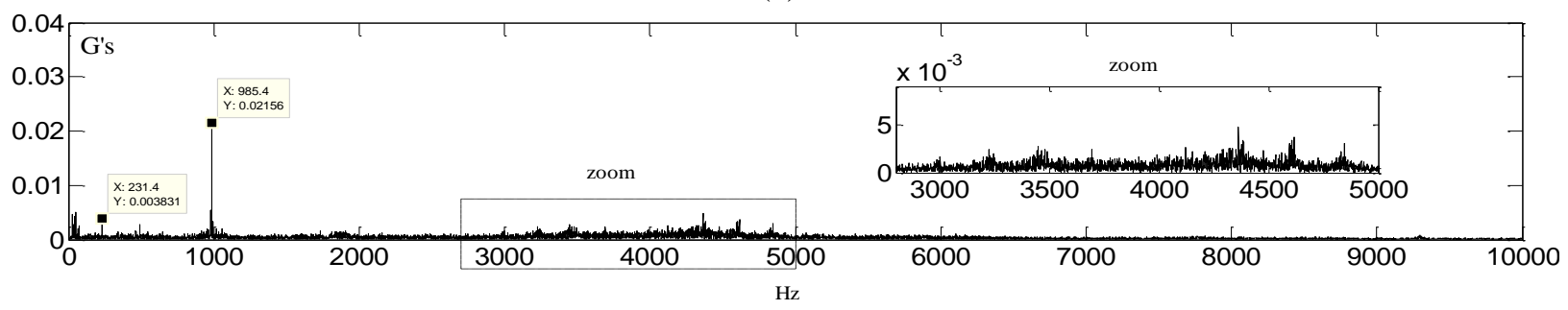

(b)

Fig. 5: Spectra of the raw signals - (a) 2004.02.16.03.02 [22] or No 531 of $T_{1}$ or No 45 of $T_{1, n}$ and (b) 2004.02.16.03.12 [22] or No 532 of $T_{1}$ or No 46 of $T_{1, n}$. 
As a conclusion of the above obtained results, the information gathered in both groups $G_{531, B P F I}$ and $G_{531, B P F O}$ correspond to a possible healthy bearing condition.

Next the sample No 532 is inserted as input to the SORDINA. The results for the frequency fields: (a) $\Delta F_{532, B P F I, 1}$, (b) $\Delta F_{532, B P F I, 2}$, (c) $\Delta F_{532, B P F I, 3}$, (d) $\Delta F_{532, B P F O, 1}$, (e) $\Delta F_{532, B P F O, 2}$ and (f) $\Delta F_{532, B P F O, 3}$ are illustrated in Figs. 11 and 12.

According to the monitoring results summarized in Fig. 12, the characteristics of the bearing fault, which are investigated in that case, can be fairly well revealed by the clear detection of the actual $\mathrm{BPFO}^{\mathrm{M}}$ and its harmonics. Moreover, it is interesting that the peaks of the maximum differences clear dominate in the testing frequency fields $\triangle F_{532, B P F O, 1}, \triangle F_{532, B P F O, 2}$ and $\triangle F_{532, B P F O, 3}$. Then, the selected frequencies $230.5 \mathrm{~Hz}, 460.9 \mathrm{~Hz}$ and $691.4 \mathrm{~Hz}$ are used to construct the group $G_{532, B P F O}$.

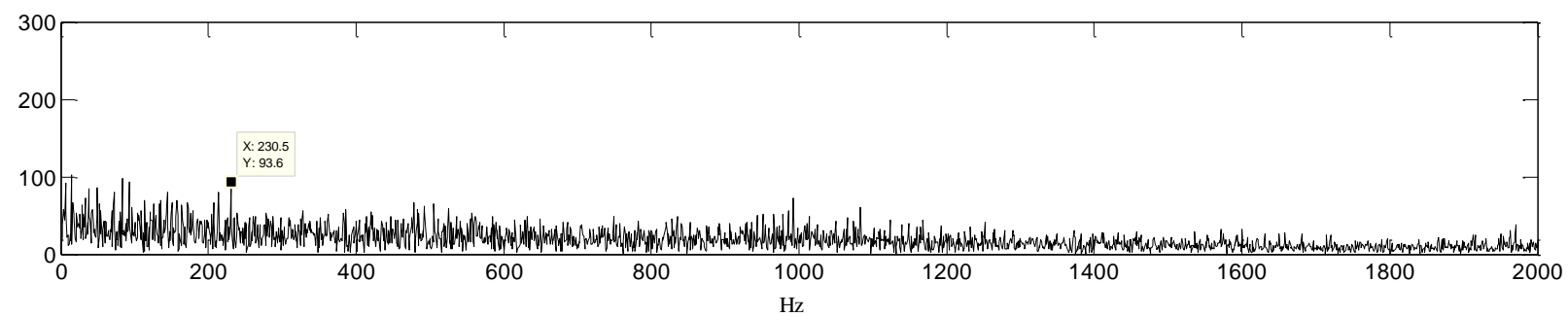

(a)

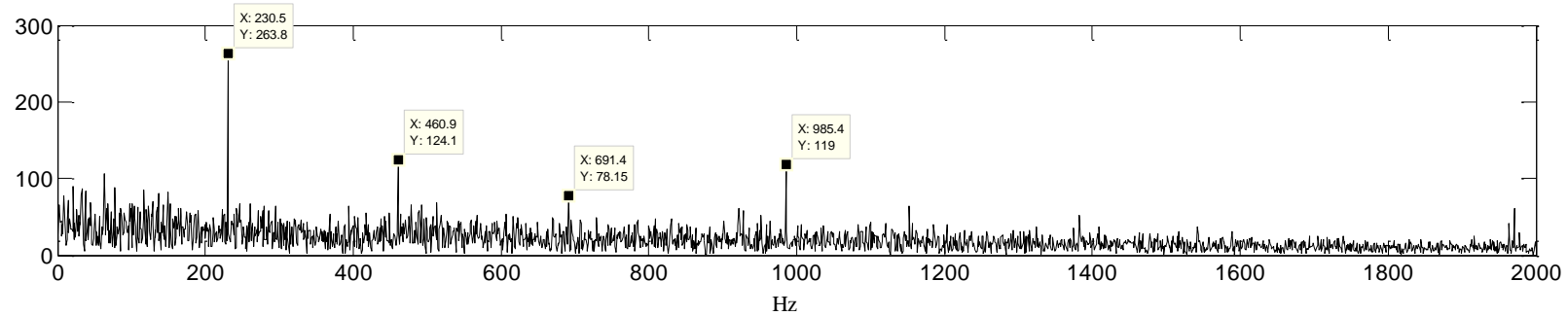

(b)

Fig. 6: Spectra of the modified signals - (a) 2004.02.16.03.02 [22] or No 531 of $T_{1}$ or No 45 of $T_{1, n}$ and (b) 2004.02.16.03.12 [22] or No 532 of $T_{1}$ or No 46 of $T_{1, n}$.

On the other hand, it can be seen at the Fig. 11 that the bearing failure does not affect the SORDINA results in the frequency fields $\Delta F_{532, B P F I, 1}, \triangle F_{532, B P F I, 2}$ and $\Delta F_{532, B P F I, 3}$. Furthermore, there is no 'harmonic link' between the selected frequencies $294.9 \mathrm{~Hz}, 591.8 \mathrm{~Hz}$ and $881.8 \mathrm{~Hz}$, which are the FCs of the $G_{532, B P F I}$. Besides, the magnitudes of the maximum differences are not dominate in the testing fields (Fig. 11). As expected, the SORDINA technique achieves to capture characteristics of a possible OR fault at its early stage.

Robustness and repeatability are desirable characteristics of a monitoring and fault diagnosis approach. In order to demonstrate the SORDINA effectiveness, the signal No 982 that experiences a severe failure stage is studied. As it can be observed in Fig. 14, the OR failure degradation leads to further magnitude domination of the maximum differences in the testing fields corresponding to OR failure: (a) $\triangle F_{982, B P F O, 1}$, (b) $\triangle F_{982, B P F O, 2}$ and (c) $\triangle F_{982, B P F O, 3}$. The selected frequency $231.4 \mathrm{~Hz}$ of the field $\triangle F_{982, B P F O, 1}$ that corresponds to the actual $\mathrm{BPFO}^{\mathrm{M}}$ differs from the one of the sample No 532 because of 
the slippage. The selected frequencies $231.4 \mathrm{~Hz}, 461.9 \mathrm{~Hz}$ and $693.4 \mathrm{~Hz}$, constituting the group $G_{982, B P F O}$, indicate the $\mathrm{BPFO}^{\mathrm{M}}$ harmonics. Under this situation, the outcomes of SORDINA, which are derived for the testing fields corresponding to IR failure: (a) $\Delta F_{982, B P F I, 1}$, (b) $\Delta F_{982, B P F I, 2}$ and (c) $\triangle F_{982, B P F I, 3}$, are relatively insensitive to the OR failure (Fig. 13).

Afterwards, for each testing modified signal $j$, the selected 9 FCs are used as MW frequency centers in the IFESIS approach, where the bandwidth $f_{b, I F}$ of the MWs is considered equal to $10 \mathrm{~d} f$. Thus, a cluster of $9 \mathrm{MW}$ is partially applied resulting to a key feature $S V_{j, T}$, called overall MSV. Firstly, the IFESIS is applied using the FCs of the group $G_{j, R P M}$. As a consequence, the feature $\sum_{i=1}^{2} S V_{j, i R P M}$ corresponding to the shaft speed harmonics is calculated. Secondly, the group $G_{j, B P F O}$ is loaded at IFESIS resulting to the feature $\sum_{i=1}^{k_{2}} S V_{j, i B P F O}$.

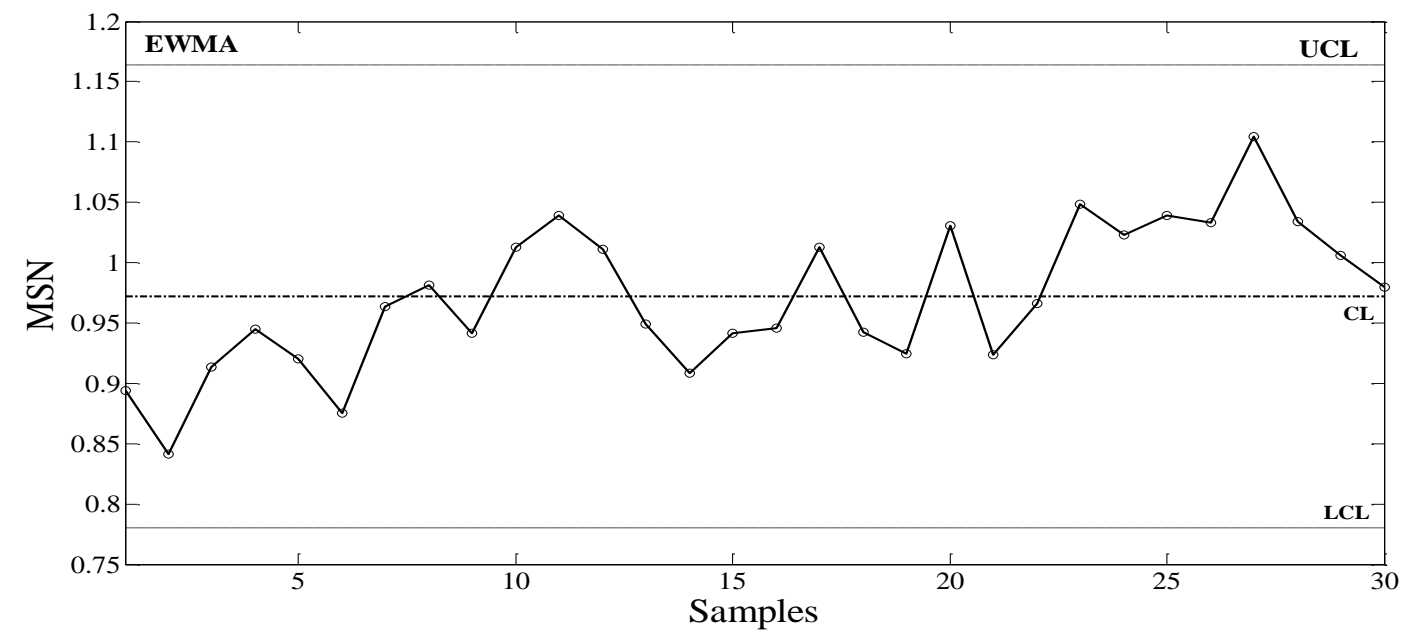

Fig. 7: Threshold UCL estimation through the EWMA model using MSVs of the training data set $S_{t r}$.

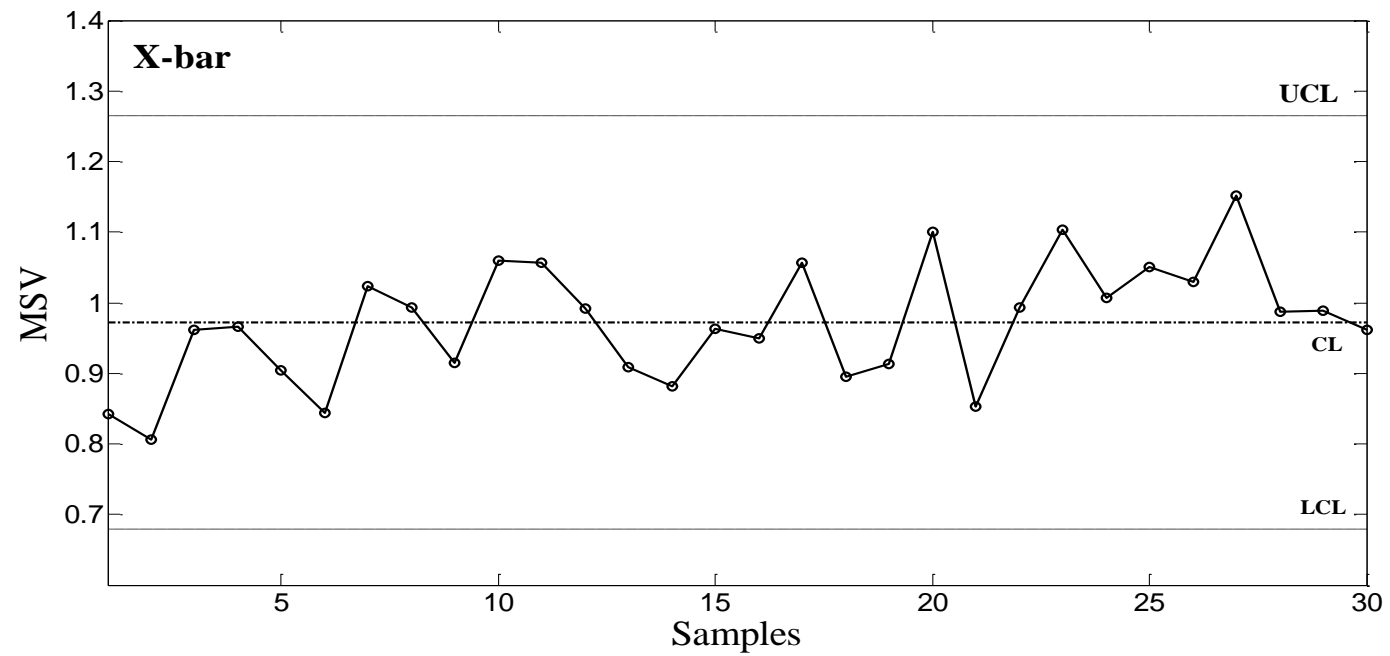

Fig. 8: Threshold UCL estimation through the X-bar model using MSVs of the training data set $S_{t r}$.

Then, the feature $\sum_{i=1}^{k_{3}} S V_{j, i B P F I}$ is calculated by the IFESIS employing the group $G_{j, B P F I}$. After these three steps, the overall feature $S V_{j, T}=\sum_{i=1}^{k_{2}} S V_{j, i B P F O}+\sum_{i=1}^{k_{3}} S V_{j, i B P F I}+\sum_{i=1}^{2} S V_{j, i R P M}$ corresponding to 
the bearing health condition (normal or abnormal) at the time $j$ is computed. In Fig. 15, the applied MWs of the IFESIS to the samples No 531, No 532 and No 982 of the first data set is presented.

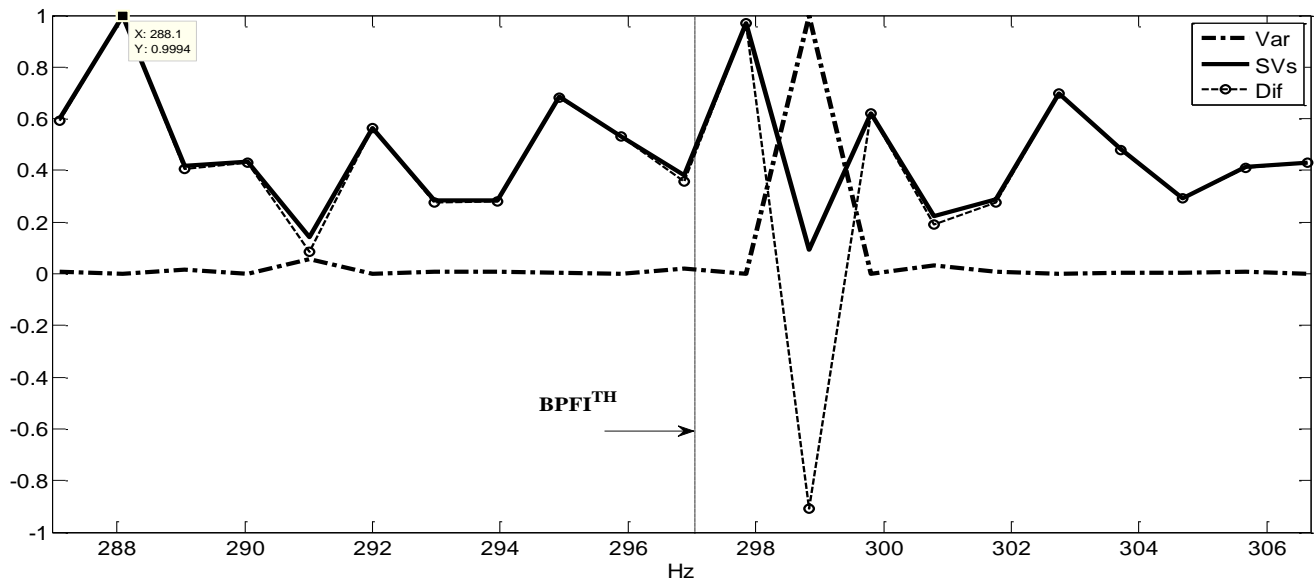

(a)

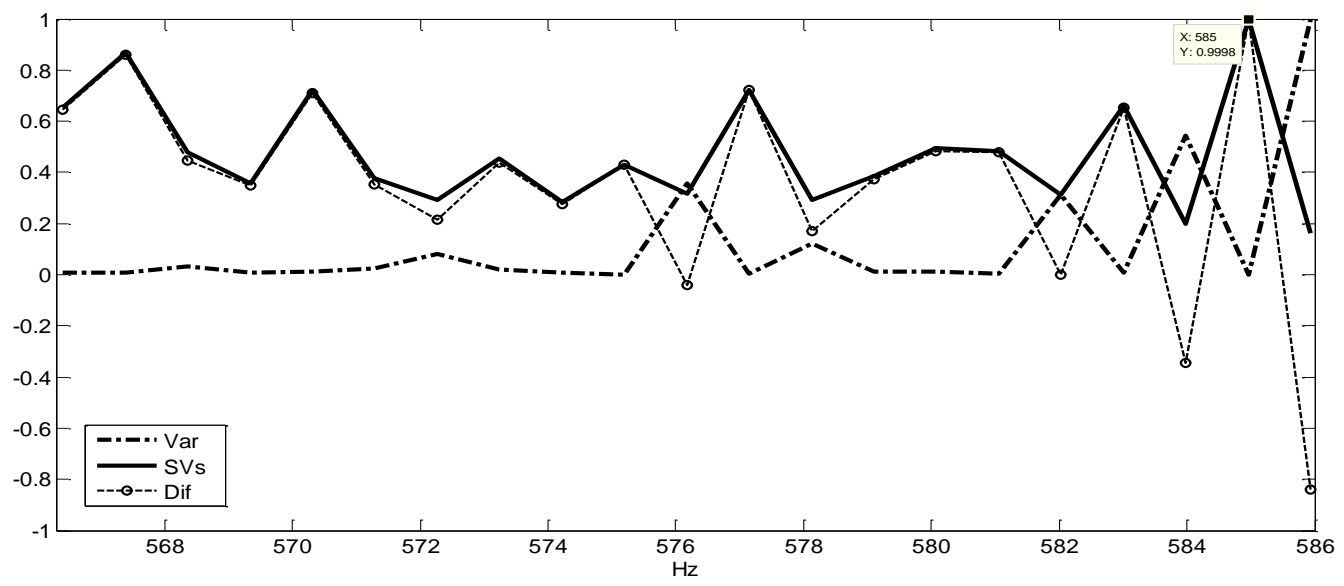

(b)

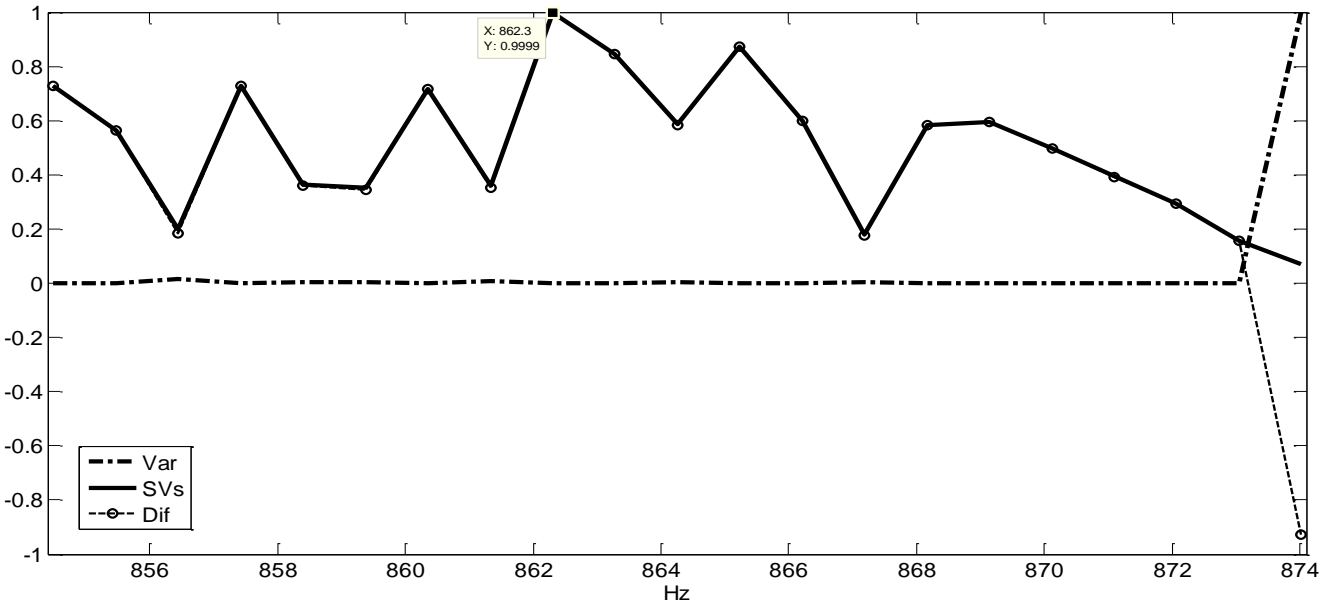

(c)

Fig. 9: SORDINA for signal No 531 - the differences of the normalized MSVs and the relative normalized IF variances for the (a) $\Delta F_{531, B P F I, 1}$, (b) $\Delta F_{531, B P F I, 2}$ and (c) $\Delta F_{531, B P F I, 3}$. 


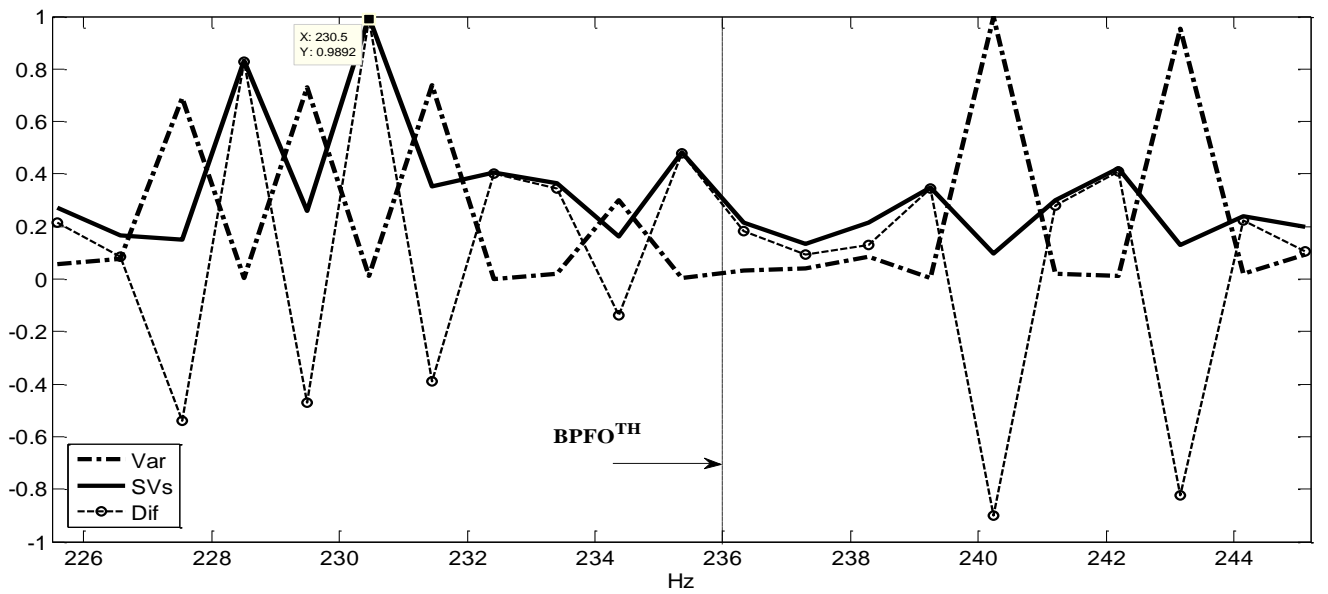

(a)

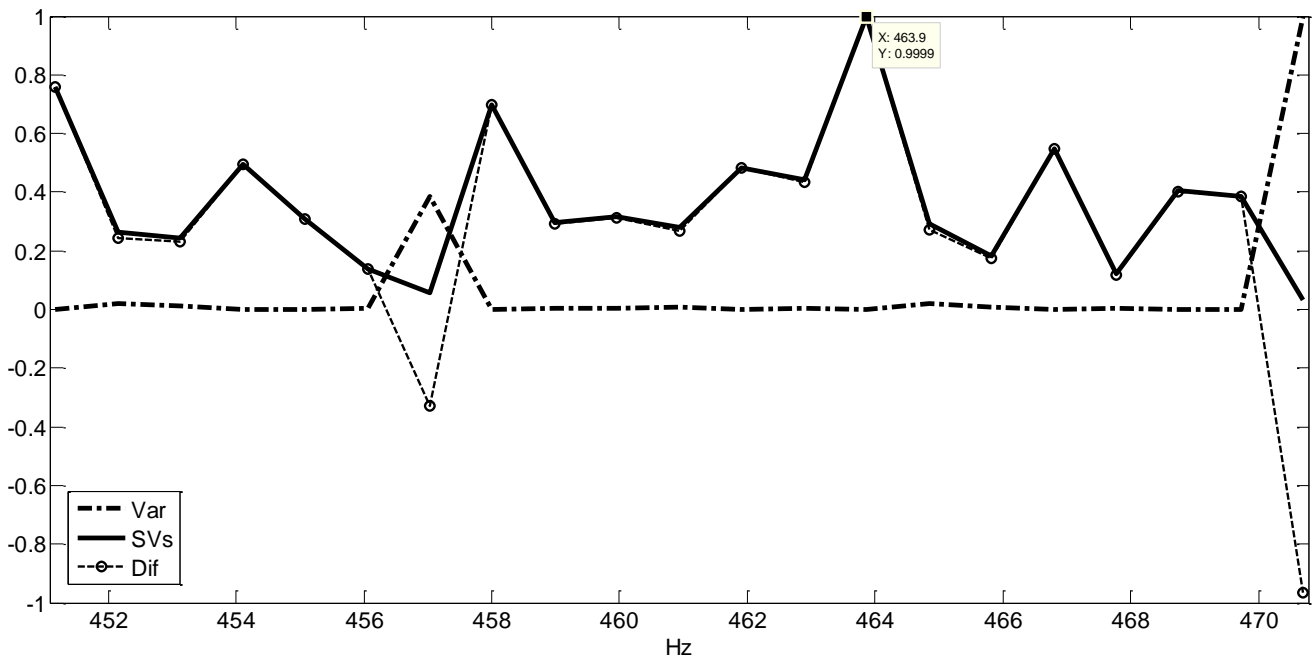

(b)

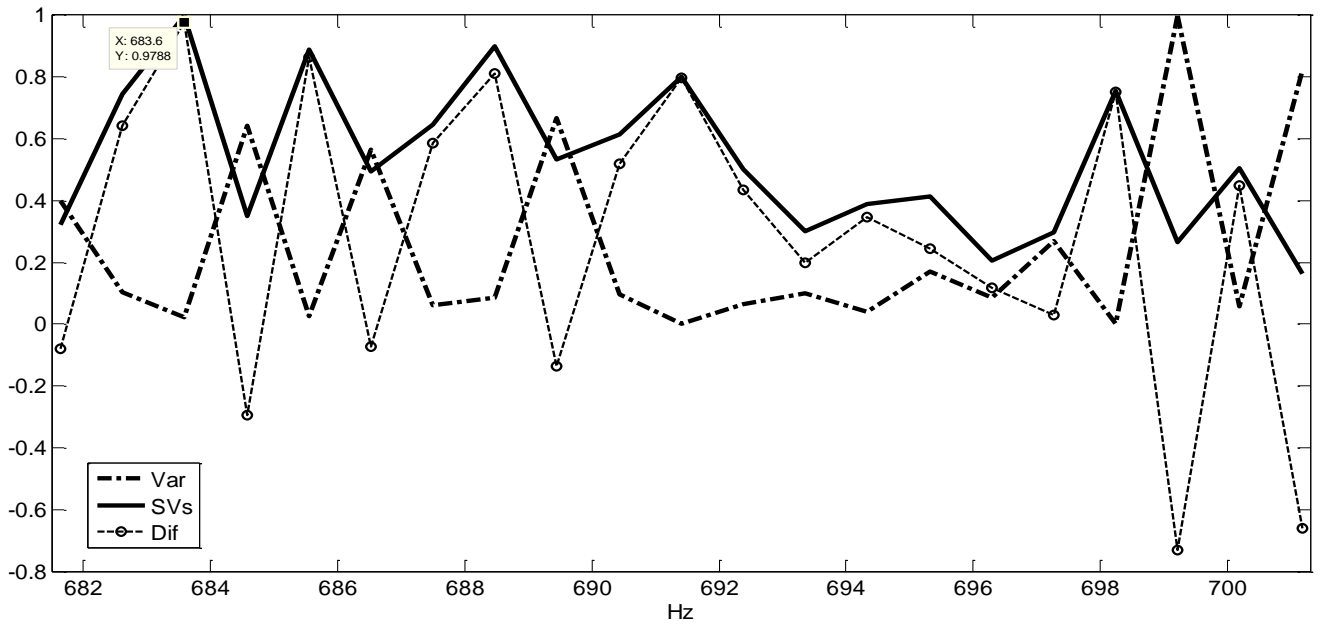

(c)

Fig. 10: SORDINA for signal No 531 - the differences of the normalized MSVs and the relative normalized IF variances for the (a) $\triangle F_{531, B P F O, 1}$, (b) $\triangle F_{531, B P F O, 2}$ and (c) $\triangle F_{531, B P F O, 3}$. 


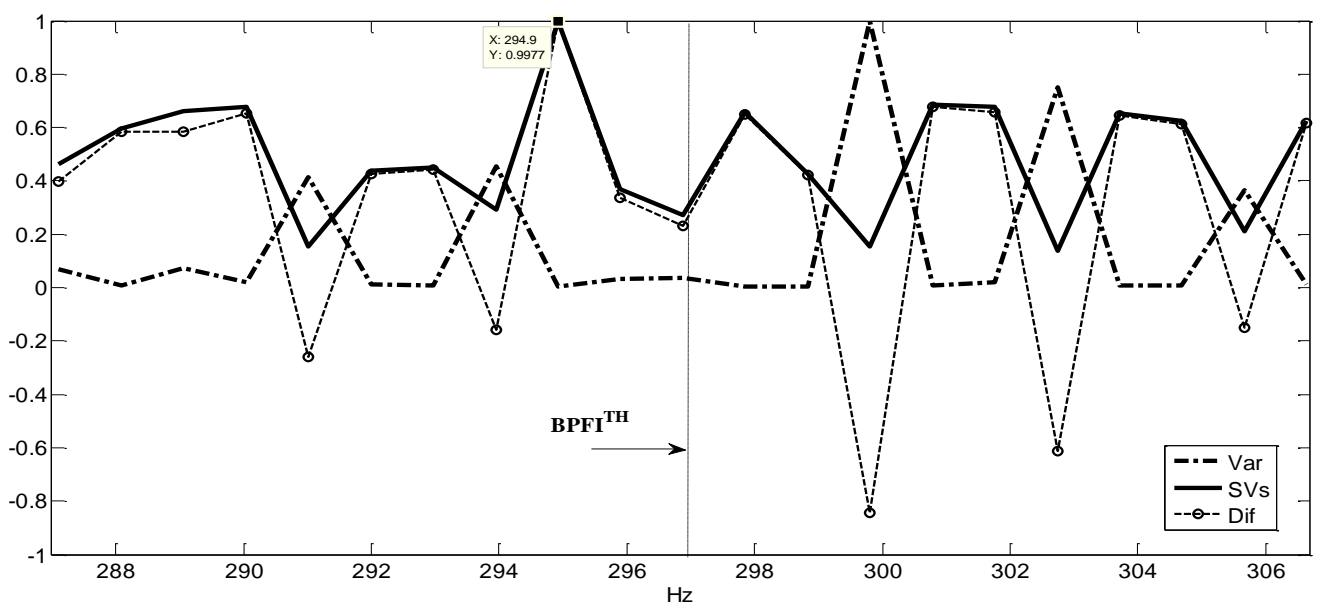

(a)

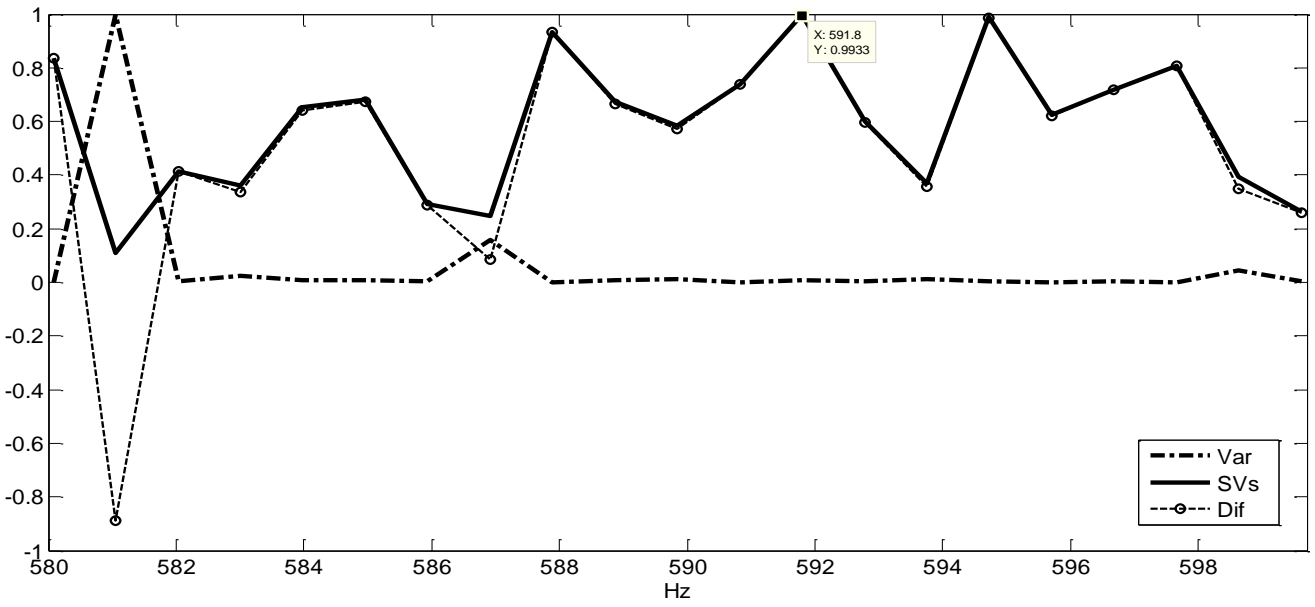

(b)

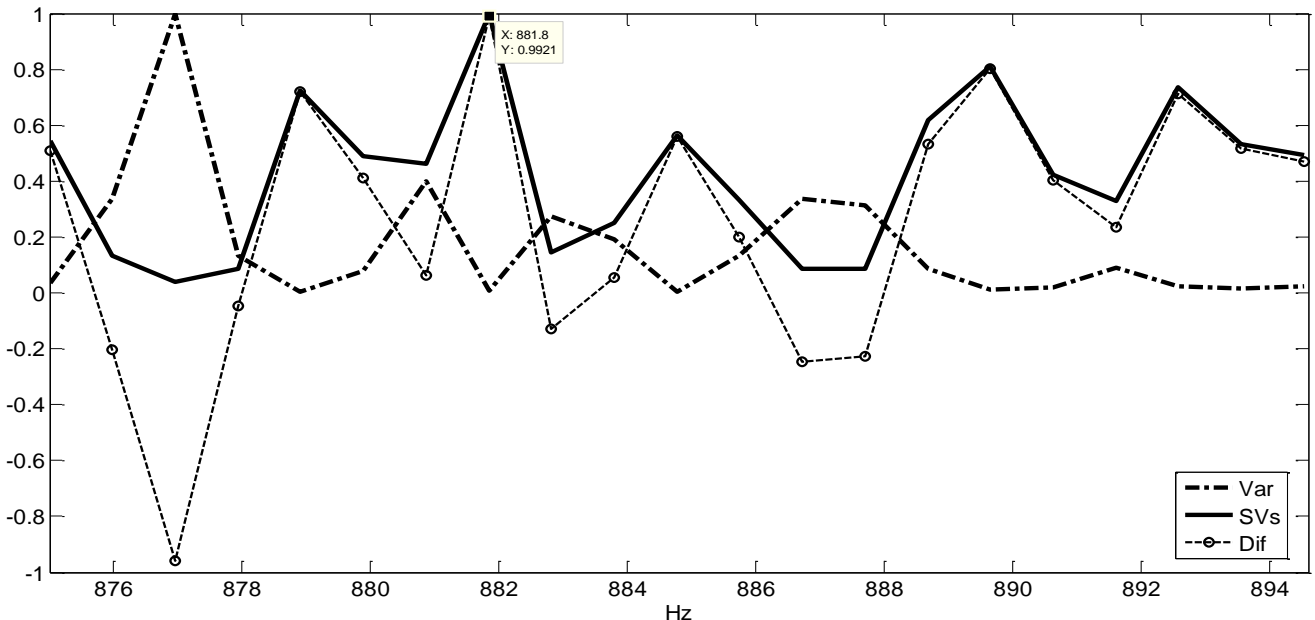

(c)

Fig. 11: SORDINA for signal No 532 - the differences of the normalized MSVs and the relative normalized IF variances for the (a) $\Delta F_{532, B P F I, 1}$, (b) $\Delta F_{532, B P F I, 2}$ and (c) $\Delta F_{532, B P F I, 3}$. 


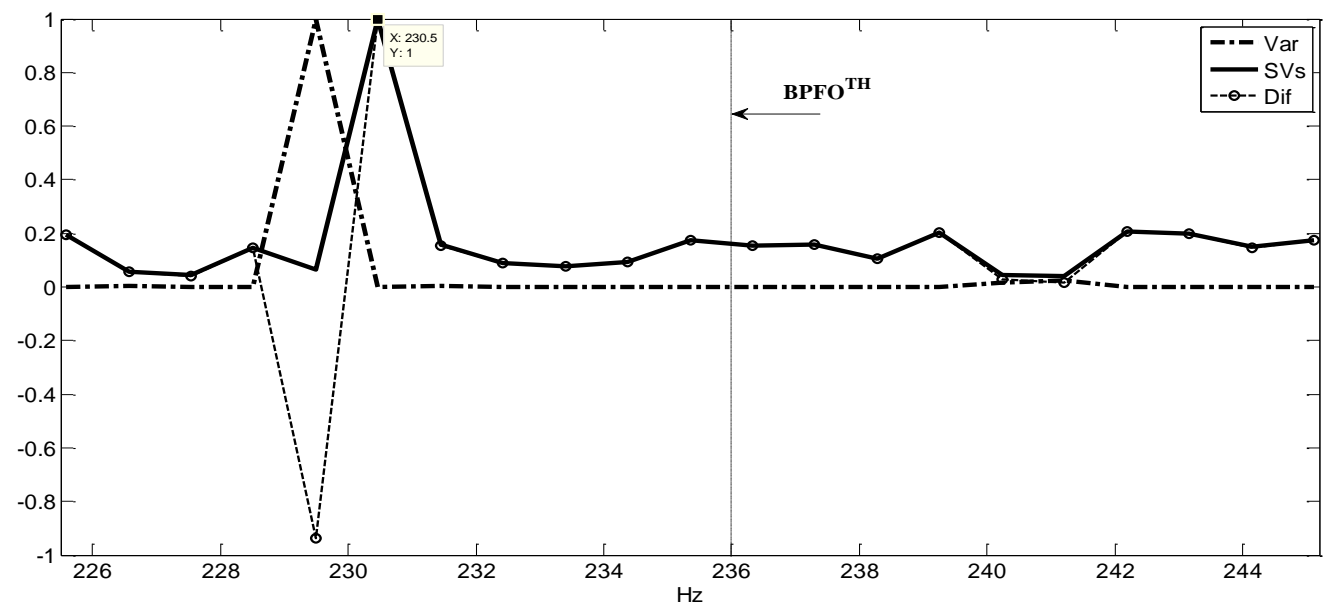

(a)

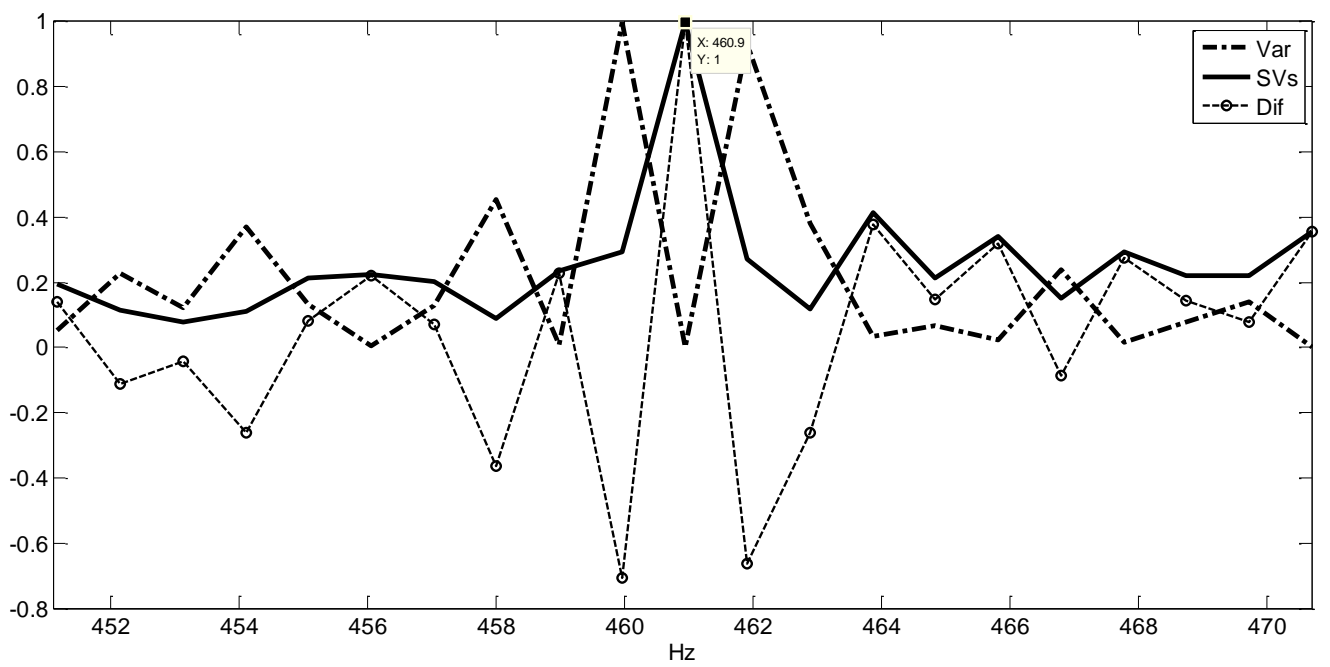

(b)

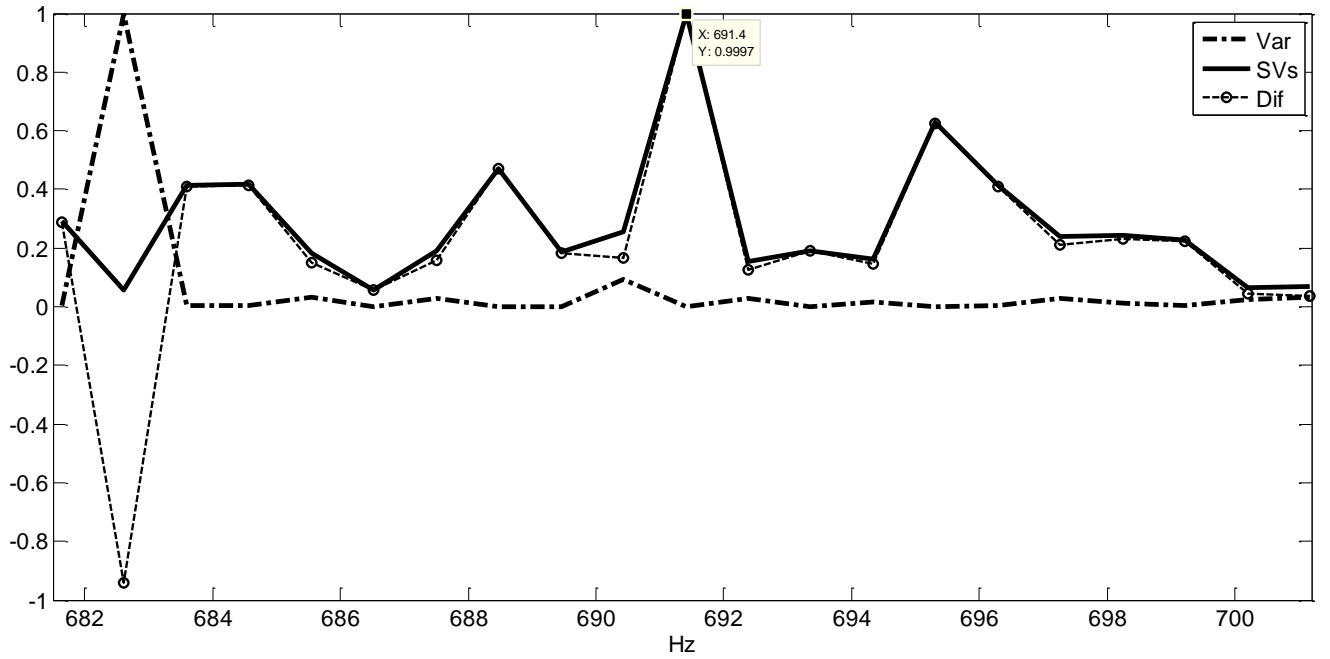

(c)

Fig. 12: SORDINA for signal No 532 - the differences of the normalized MSVs and the relative normalized IF variances for the (a) $\triangle F_{532, B P F O, 1}$, (b) $\triangle F_{532, B P F O, 2}$ and (c) $\triangle F_{532, B P F O, 3}$. 


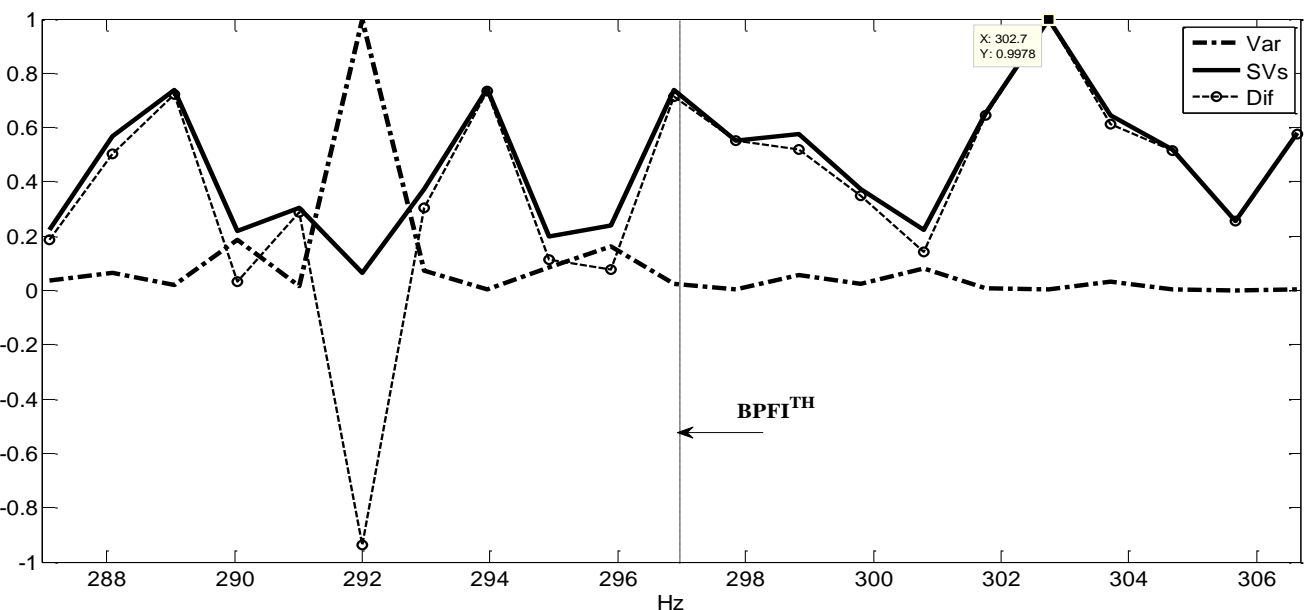

(a)

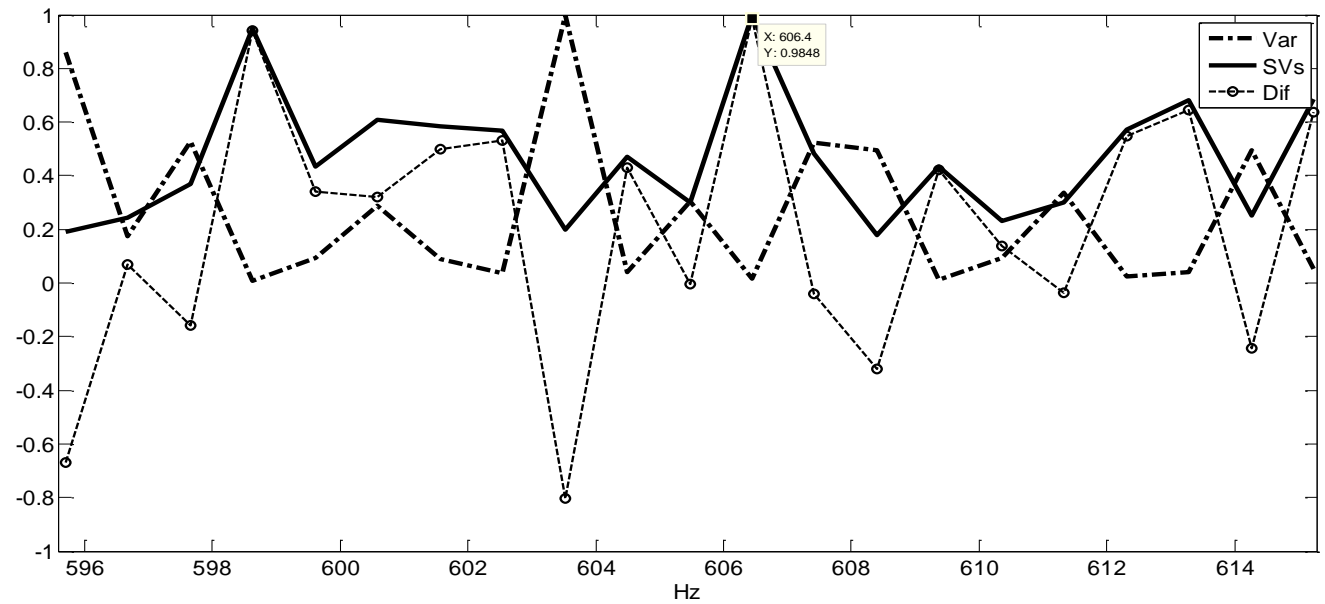

(b)

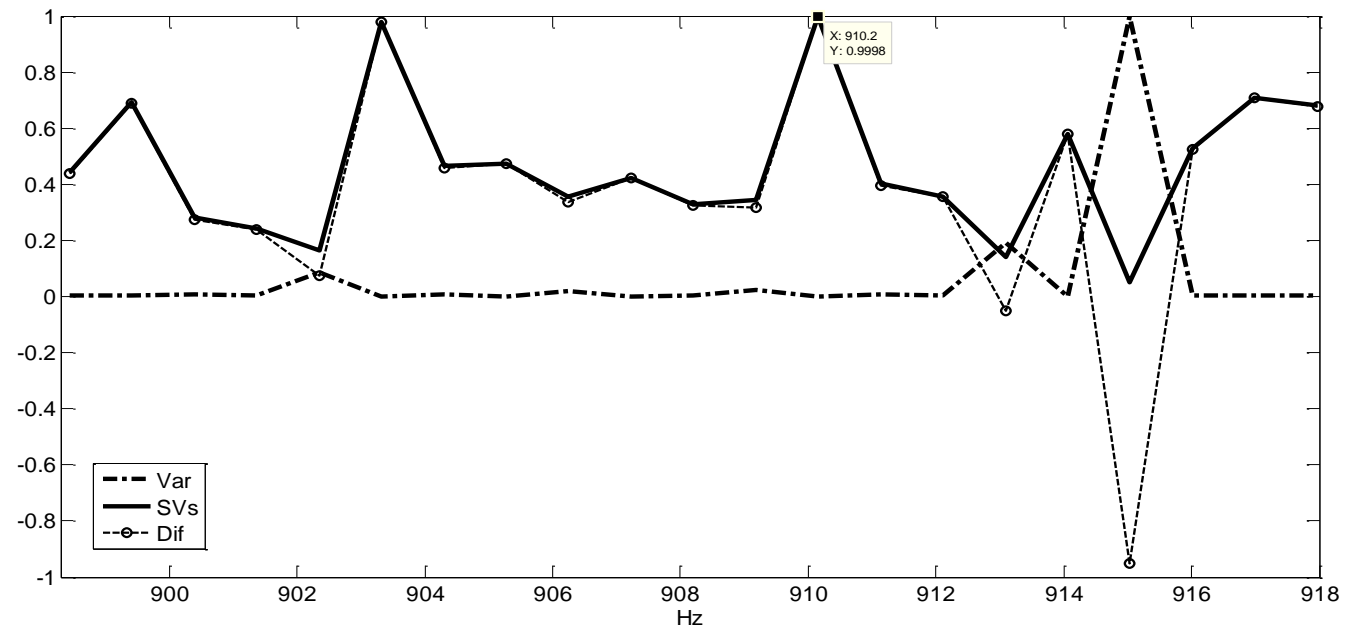

(c)

Fig. 13: SORDINA for signal No 982 - the differences of the normalized MSVs and the relative normalized IF variances for the (a) $\Delta F_{982, B P F I, 1}$, (b) $\Delta F_{982, B P F I, 2}$ and (c) $\Delta F_{982, B P F I, 3}$. 


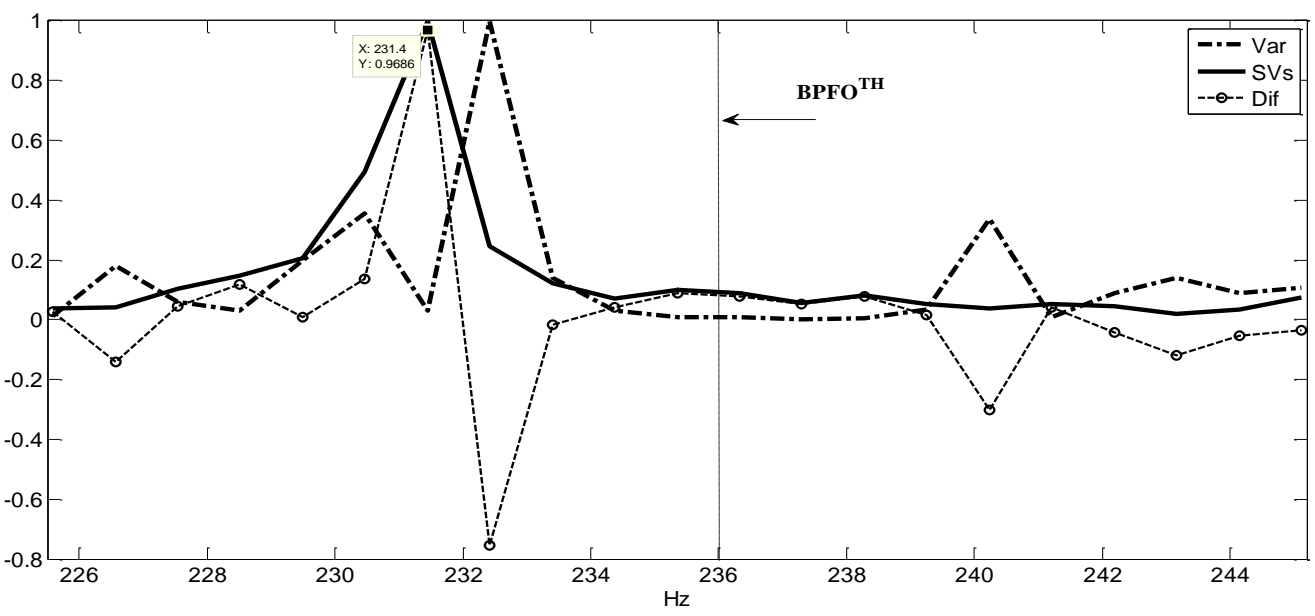

(a)

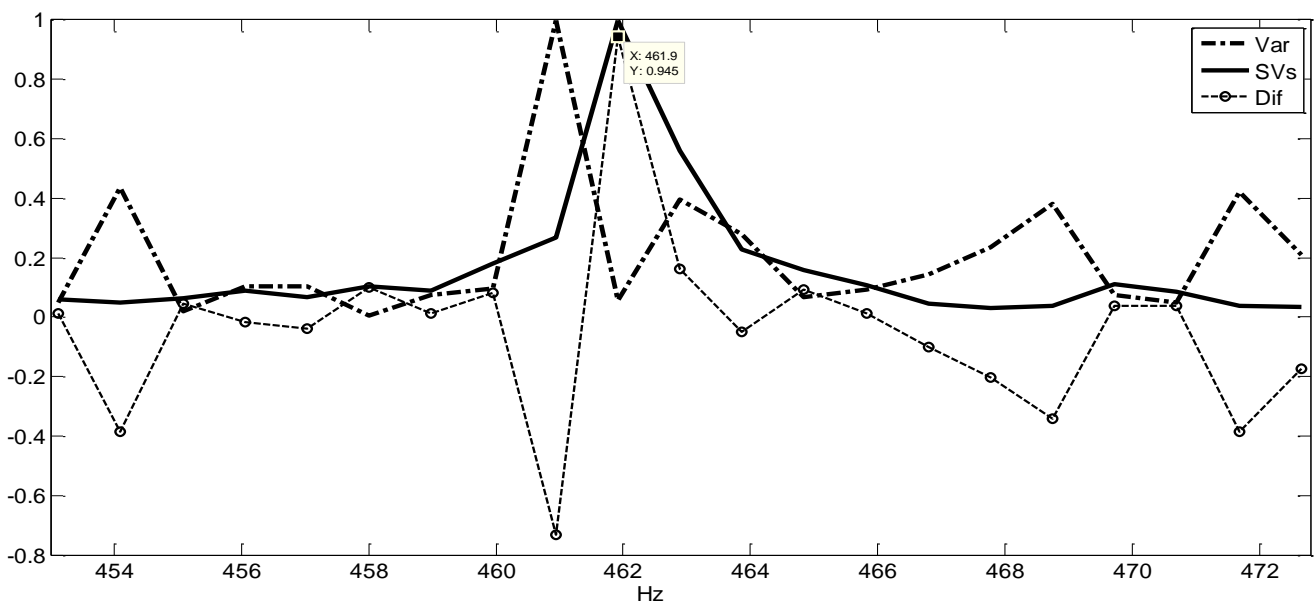

(b)

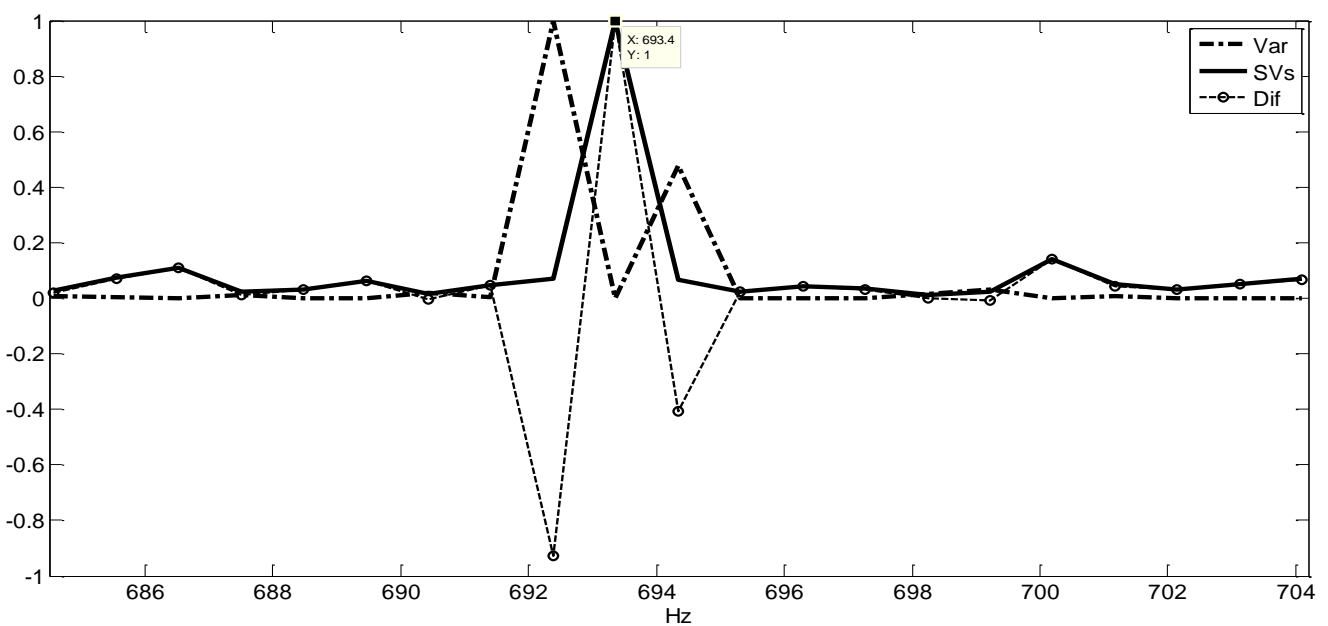

(c)

Fig. 14: SORDINA for signal No 982 - the differences of the normalized MSVs and the relative normalized IF variances for the (a) $\triangle F_{982, B P F O, 1}$, (b) $\triangle F_{982, B P F O, 2}$ and (c) $\triangle F_{982, B P F O, 3}$. 


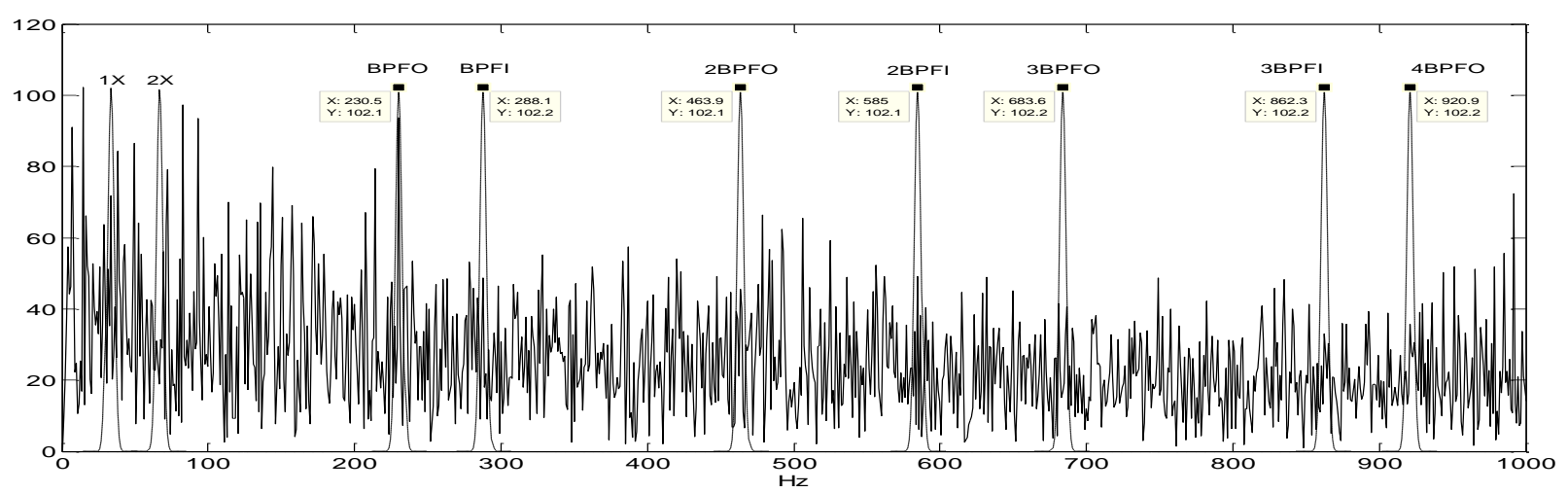

(a)

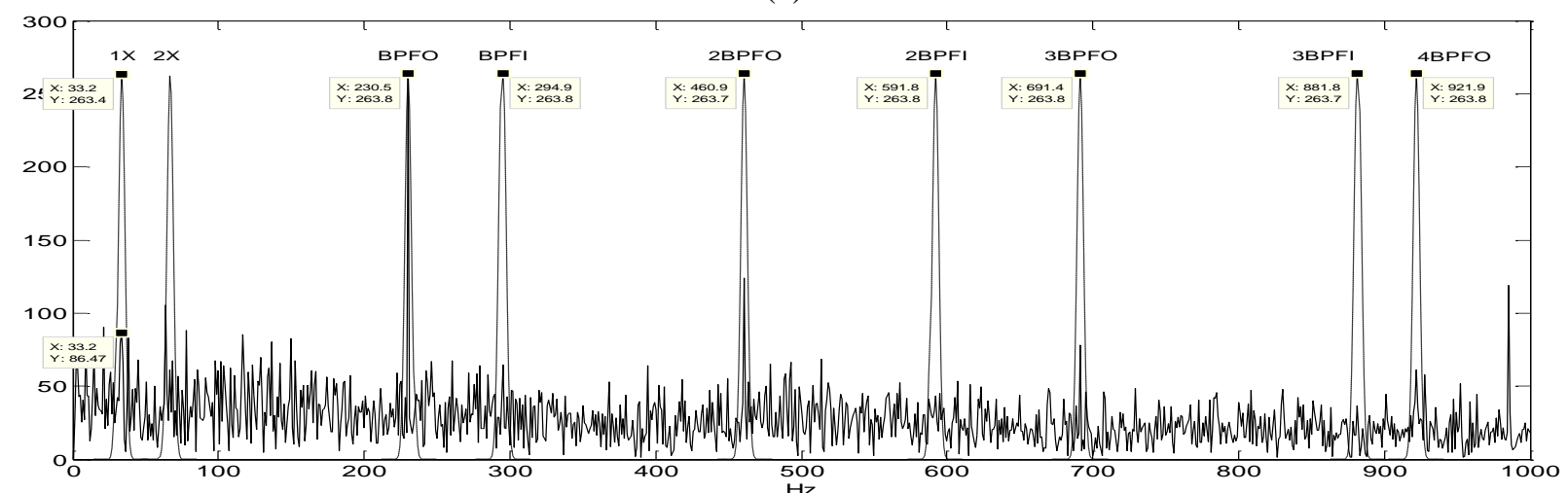

(b)

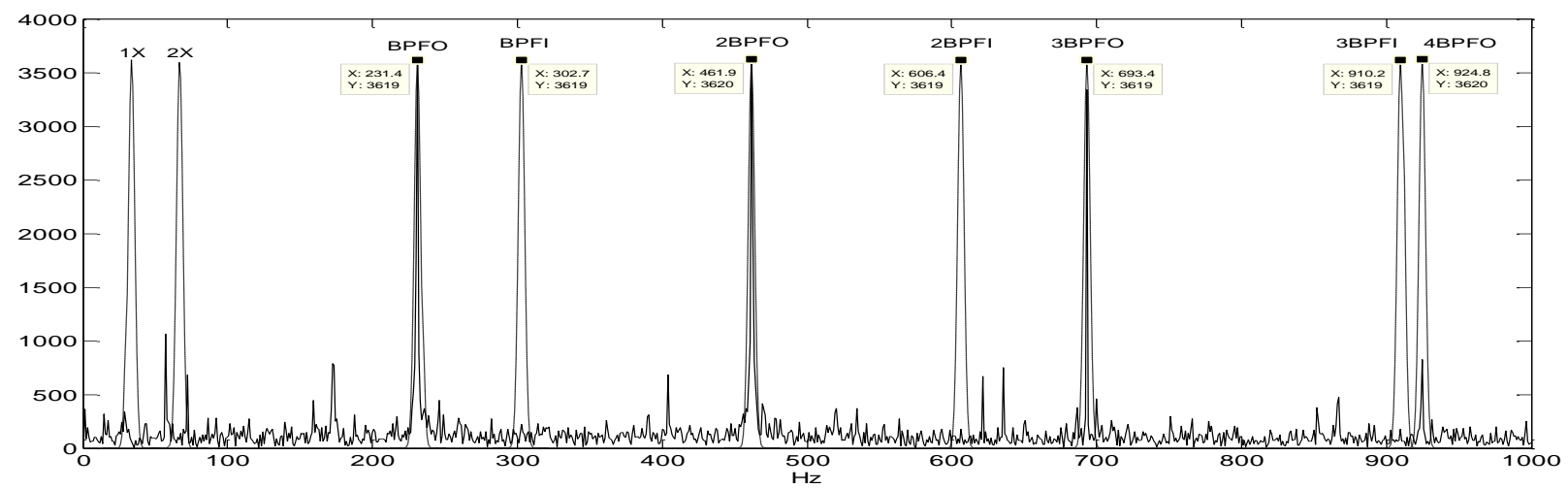

(c)

Fig. 15: IFESIS - a cluster of 9 Morlet wavelets is partially applied to the spectrum of the shape modified signals (a) No 531, (b) No 532 and (c) No 982.

Some of the raw SV curves derived by the IFESIS for the shape modified samples No 531, No 532 and No 982 utilizing the groups $G_{j, B P F O}$ and $G_{j, B P F I}$ are depicted in Fig. 16, demonstrating the corresponding patterns and magnitude levels to the normal and the abnormal operating conditions.

For a signal captured under normal operating conditions, the results in Fig. 16(a) show that the SV curves corresponding to $\mathrm{BPFO}^{\mathrm{M}}$ and $\mathrm{BPFI}^{\mathrm{M}}$ are close. Additionally, these SVs present small magnitude values similar to those of noise.

Besides, as presented in Fig. 16(b), at the very first slight failure stage, the amplitude of the SV curve corresponding to $\mathrm{BPFO}^{\mathrm{M}}$ increases against the one of the $\mathrm{SV}$ curve corresponding to $\mathrm{BPFI}^{\mathrm{M}}$ that remains almost invariable. This is due to the development of an OR wear. Bearing failure degradation (sample No 
982) leads to further magnitude deviation between the above compared SV curves as it can be observed in Fig. 16(c). Similarly, the corresponding SV curves for the rest of the harmonics of the groups $\mathrm{G}_{\mathrm{j}, \mathrm{BPFO}}$ and $\mathrm{G}_{\mathrm{j}, \mathrm{BPFI}}$ are analogous. Based on the aforementioned results, it can be concluded that the proposed features are able to follow effectively and quantify the operating condition of the machine.

Following the application of the novel feature extraction approach to compute the key feature $S V_{j, T}$ of the testing signal $j$, the 'abnormal detection' module is triggered in order to adapt the estimated feature in a EWMA or X-bar basis and further classify it. The feature is classified as normal whenever it is located below the estimated UCL threshold.

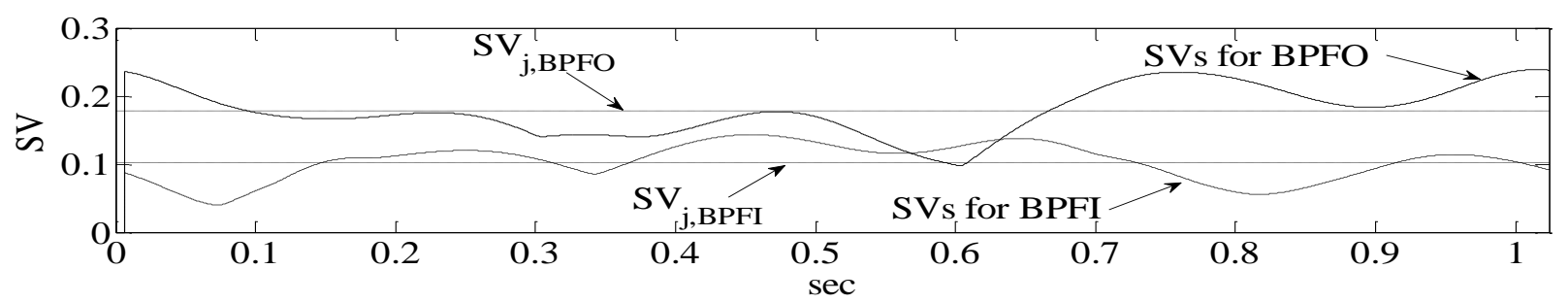

(a)

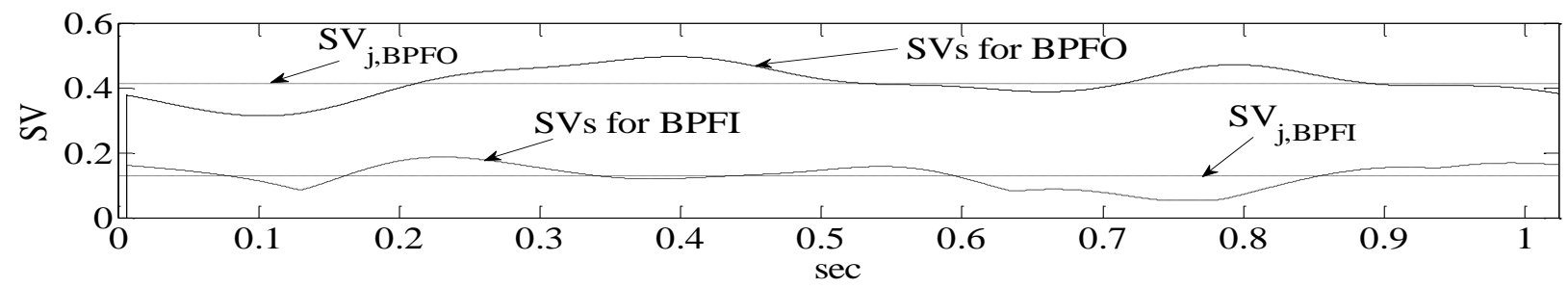

(b)

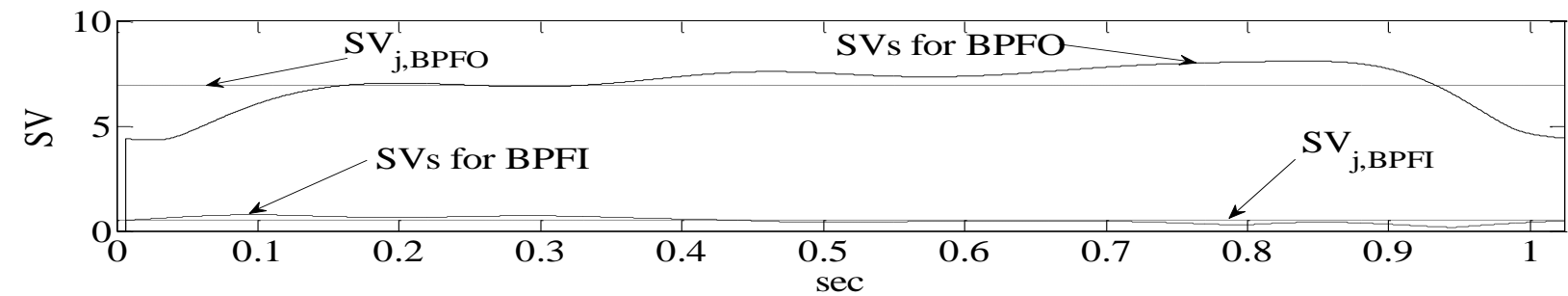

(c)

Fig. 16: IFESIS - calculated SV curves for the shape modified signals (a) No 531, (b) No 532 and (c) No 982.

The performance of the proposed hybrid method for the data sets $S_{1}$ and $S_{2}$ is demonstrated in the Figs. 16 and 17 , respectively.

Moreover, the influence of the weight $c$ on EWMA model is studied for different values between 0 and 1. The performance of EWMA using MSVs in accordance to the weight $c$ is presented in Table 1. Higher values of the weight factor $c$ improve the efficiency of the statistical model. The EWMA model starts to behave like an X-bar model for values close to 1. Moreover, the performance of the EWMA model remains almost unchanged for values of the weight factor $c$ that range between 0.3 and 0.8 . As a consequence, the weight factor $c$ is proposed to be selected equal to 0.6.

In the case study of the data set $S_{1}$, using both control charts basis, the proposed method efficiently monitors and detects changes in a dynamic operating condition misclassifying only the very first faulty signal as healthy. However, when the overall MSV feature corresponds to normal bearing behavior, the 
prediction error of the proposed model is zero. The accuracy of the bearing behavior identification is presented in Fig 17. The consistent degradation pattern of the overall MSV feature is shown in Fig. 17. Likewise, in the case study of the data set $S_{2}$, the novel method demonstrates a promising outlook misclassifying as faulty only one and three healthy signals respectively using the X-bar and the EWMA control chart. A limited number of missed detection is expected as it is very difficult to accurately assess the bearing performance degradation states by using a single-feature-based model. As can be seen in Fig. 18 , the sequence of the overall MSV features constructs a bearing performance curve that is consistent to the prediction of the bearing health condition.

In general the X-bar-based training performs better than the EWMA-based training. The results in both cases indicate that the proposed feature $S V_{j, T}$ is a trustworthy diagnostic index monitoring accurately the operating condition (normal or abnormal) of a bearing.

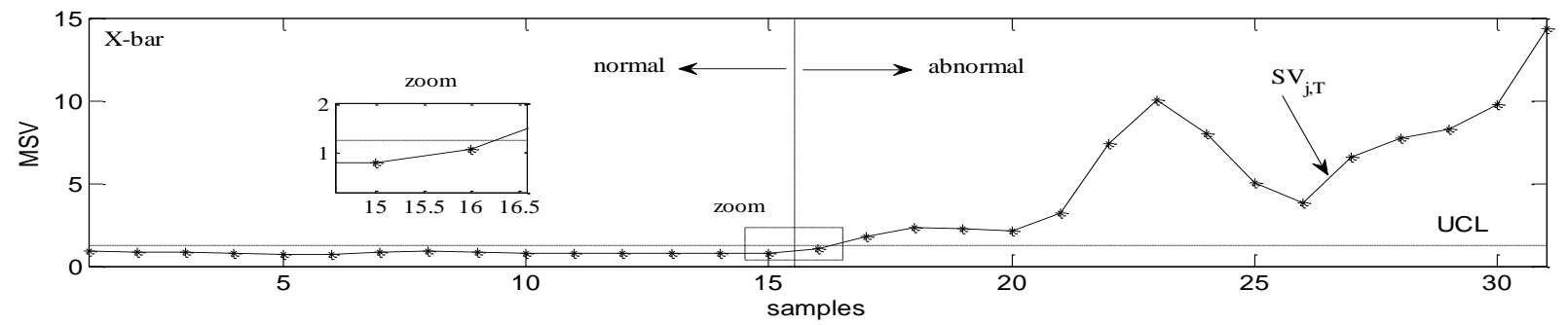

(a)

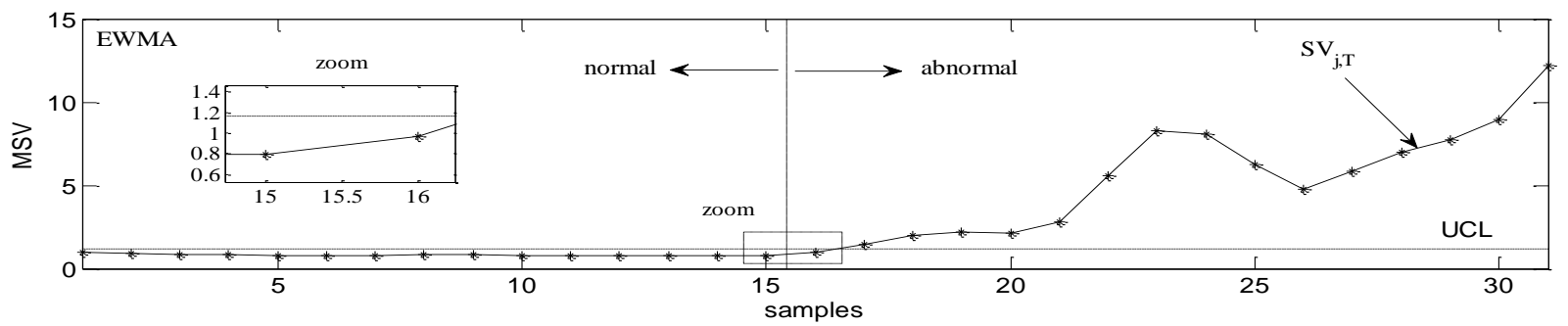

(b)

Fig. 17: Novelty detection of testing set $S_{1}$ using (a) X-bar and (b) EWMA control charts (c=0.6), trained with overall MSVs.

\subsection{Fault taxonomy}

In case the 'abnormal detection' module captures a testing feature of a fault, the 'fault classification' module is activated. Fig. 19 presents the classification results for both data sets $S_{1}$ and $S_{2}$. The vertical dashed line separates the normal and abnormal bearing states.

Unlike other typical features (RMS, CF, etc), the MSV feature has the advantage to be able to categorize the fault of a bearing. By comparing the cumulative MSVs, $S V_{j, O R}$ and $S V_{j, I R}$, corresponding to OR and IR defects, respectively, it's possible to identify the failure mode. If the $S V_{j, I R}$ is higher than the $S V_{j, O R}$ then the fault is considered to be at the IR of the bearing, otherwise the fault is considered to be at the OR. As it is observed in Fig. 18, in both data sets $S_{1}$ and $S_{2}$, the cumulative MSV features achieve to identify the fault mechanism from the very first faulty signal maintaining a considerable gap between the two compared MSVs.

As shown in Fig. 17, the 'abnormal detection' module captures a fault at the $18^{\text {th }}$ successive testing measurement. At this point, the 'taxonomy module' based on the fact that the $S V_{17, O R}$ is larger than $S V_{17, I R}$ 
recognizes an OR fault (Fig. 19(a)). As the OR fault deteriorates, the $S V_{j, \mathrm{O} R}$ is increased, whereas the $S V_{j, I R}$ is not affected by the OR fault, confirming the reliability of the proposed methodology. It is also interesting to note that in the data set $S_{1}$, even though the first faulty signal is misclassified as healthy (Fig. 17), the rise of the $S V_{j, \mathrm{O} R}$ feature is obvious (Fig. 19 (a)). According to the results presented in Fig. 18, a fault is detected at the $11^{\text {th }}$ successive testing measurement. As it is observed in Fig. 19(b), the $S V_{11, \mathrm{IR}}$ is larger than the $S V_{11, \mathrm{OR}}$ indicating the presence of an IR fault. In contrast to the $S V_{\mathrm{j}, \mathrm{O} R}$, the feature $S V_{j, I R}$ continues to rise following the IR failure deterioration.

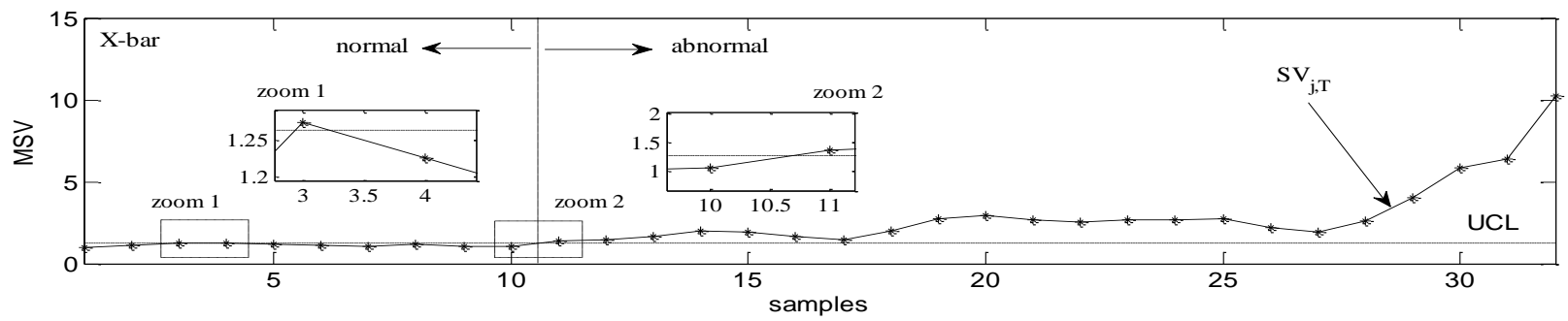

(a)

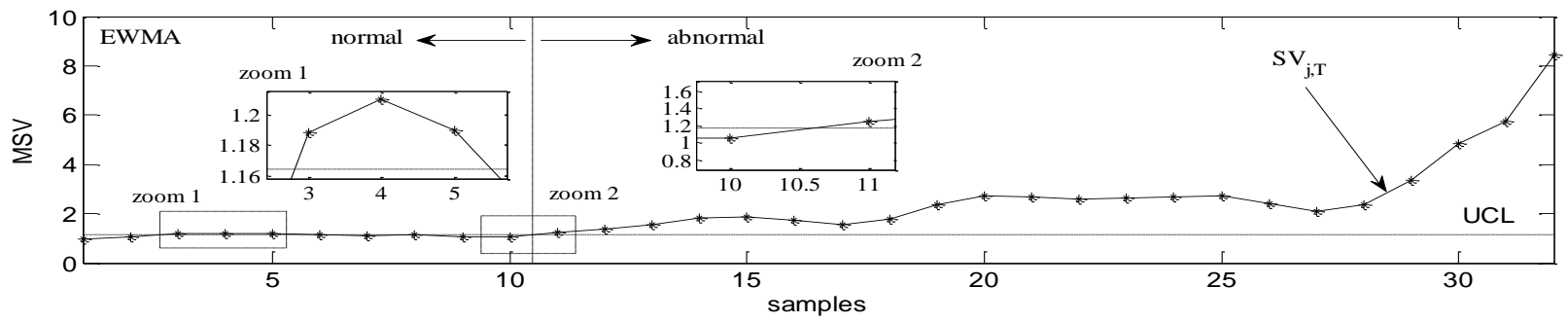

(b)

Fig. 18: Novelty detection of the testing set $S_{2}$ using (a) X-bar and (b) EWMA control charts ( $c=0.6$, trained with overall MSVs.

Table 1

Performance of the EWMA chart in accordance to the weight factor $c$ using MSV

\begin{tabular}{cccccccccc}
\hline & $\mathrm{c}$ & \multicolumn{2}{c}{0.1} & \multicolumn{2}{c}{0.3} & & 0.6 & \multicolumn{3}{c}{0.8} \\
\hline & $\mathrm{S}_{1}$ & $\mathrm{~S}_{2}$ & $\mathrm{~S}_{1}$ & $\mathrm{~S}_{2}$ & $\mathrm{~S}_{1}$ & $\mathrm{~S}_{2}$ & $\mathrm{~S}_{1}$ & $\mathrm{~S}_{2}$ \\
\cline { 2 - 10 } false alarm & 0 & 8 & 0 & 7 & 0 & 3 & 0 & 2 \\
missed detection & 2 & 0 & 1 & 0 & 1 & 0 & 1 & 0 \\
delay & 2 & 0 & 1 & 0 & 1 & 0 & 0 & 0 \\
accuracy rate (\%) & 93.5 & 75.0 & 96.8 & 78.1 & 96.8 & 90.6 & 96.8 & 93.8 \\
\hline
\end{tabular}

\subsection{Comparative Study}

In order to better evaluate the effectiveness of the proposed hybrid method, the typical features KU, RMS, $\mathrm{CF}$ and SF are calculated for all the signals in: (a) raw form (RF), (b) shape modified form (SMF) and (c) envelope form using the kurtogram (ENKU).

The UCL thresholds for each one of the RF, SMF and ENKU features are calculated through the X-barbased and EWMA-based training procedures. The first 30 signals of the data sets $T_{1, n}$ and $T_{2, n}$ are used in the training process. $18 \mathrm{UCL}_{\mathrm{X} \text {-bar }}$ and $18 \mathrm{UCL}_{\mathrm{EWMA}}$ thresholds are estimated. After the complete of the 
training procedure the remaining 31 and 32 signals of the testing sets $S_{1}$ and $S_{2}$ are adapted and tested according to the statistical methods used. Some representative results of the testing process are illustrated in the Figs. 20 - 28 and in the Tables 2 - 6. The sensitivity of the features is quantified by calculating the false alarms, the missed detections, the fault detection delay in samples and the accuracy rates of the method performance (\%). It should be noted that a false alarm is the erroneous fault report during the normal operation of a bearing, while a missed detection corresponds to a failure of the fault detector to identify an abnormal situation.

Although, as shown in Tables 2, 3, 5 and 6 the CF abnormal detector fails completely to track the anomalous situations in the testing case of data set $S_{1}$, it performs almost excellent in the case of $S_{2}$, misclassifying as healthy only the very first faulty signal using both the X-bar and EWMA control charts (Fig 20).

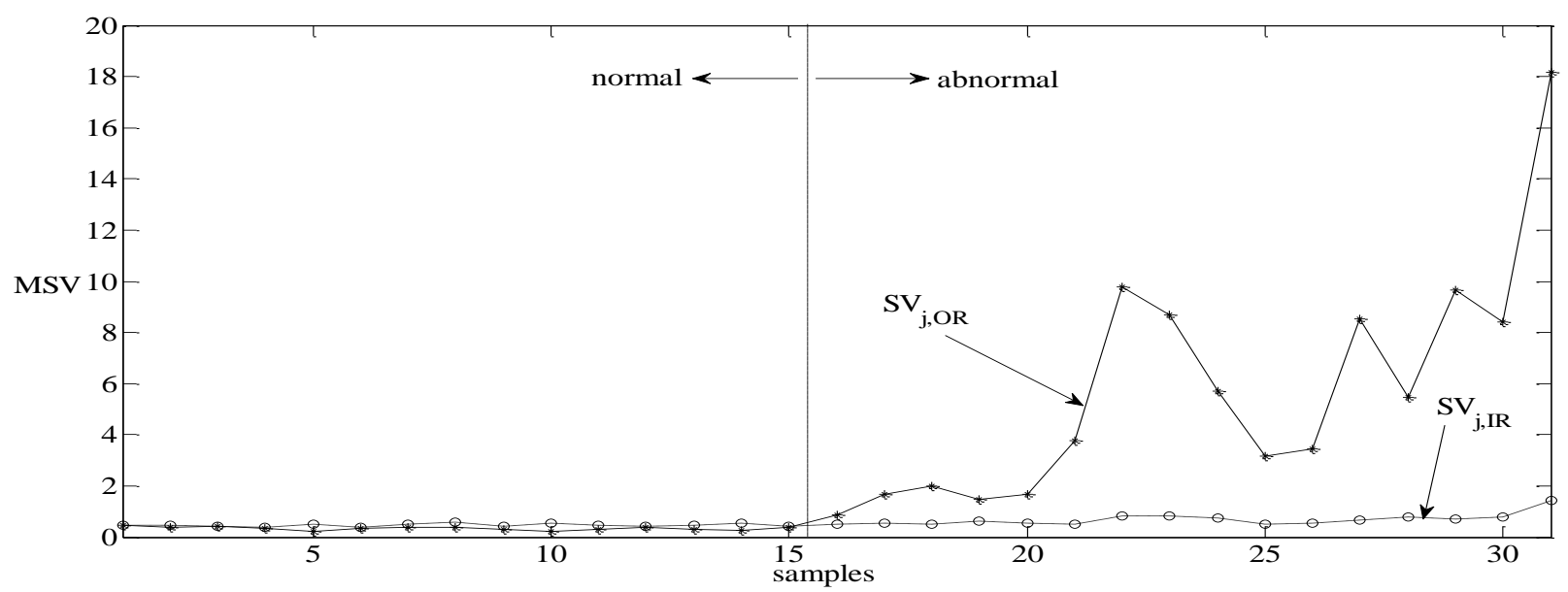

(a)

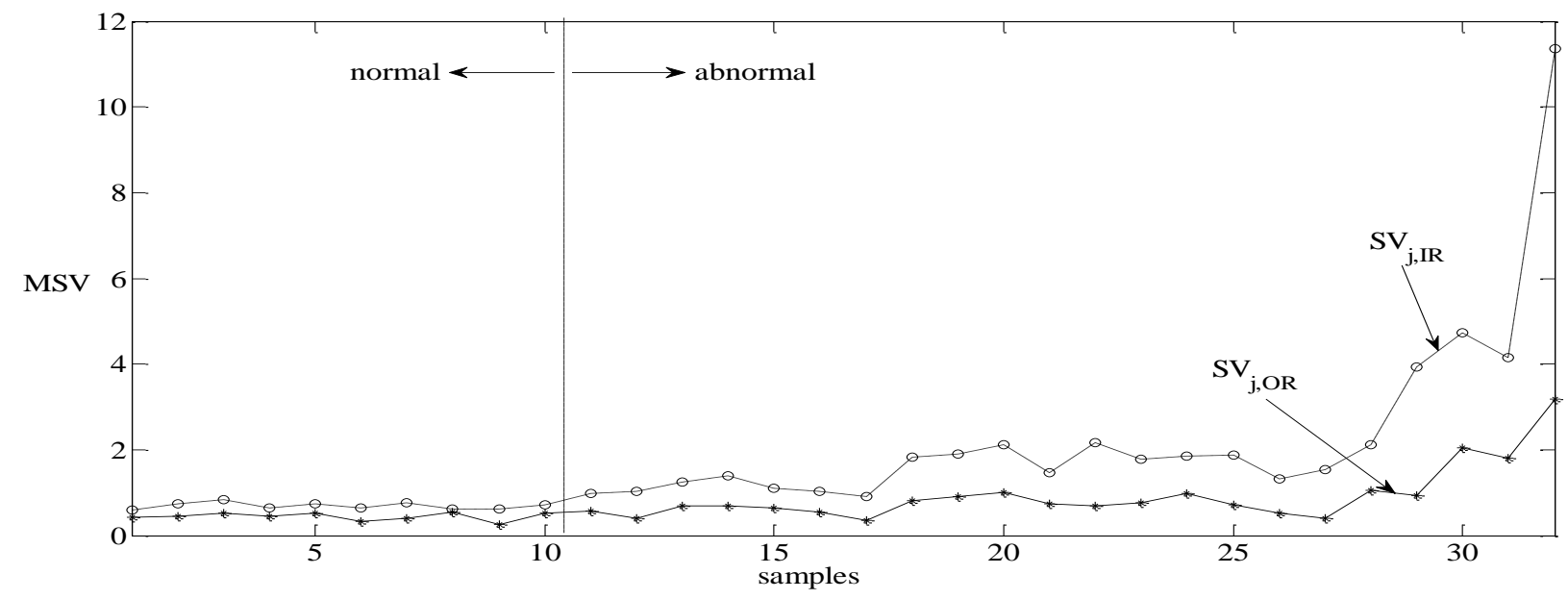

(b)

Fig. 19: Fault classification results for the testing sets (a) $S_{1}$ and (b) $S_{2}$.

As shown in Fig. 21 and Tables 2 and 3, the RMS features extracted by the raw data and calculated using both the X-bar-based and the EWMA-based procedures, achieve to detect both bearing failures only 
towards the very end. On the other hand, as shown in Tables 5 and 6, the performance of both statistical models is increased for the data set $S_{2}$ when features extracted by data in ENKU are used.

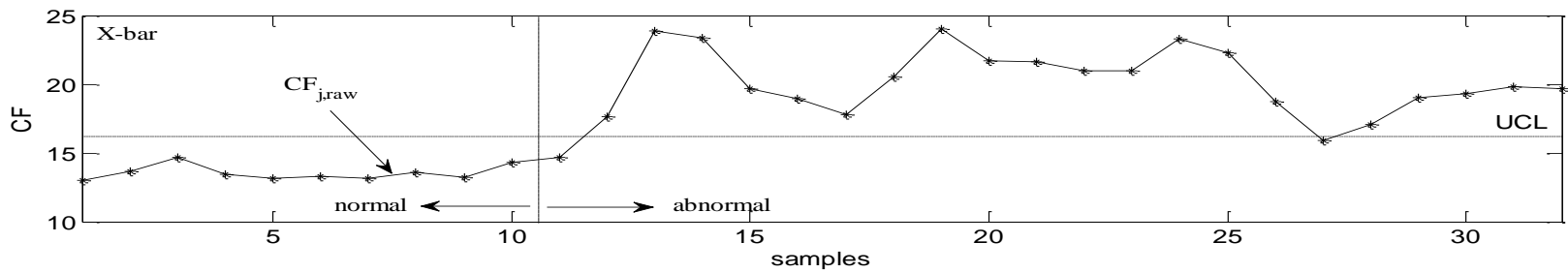

(a)

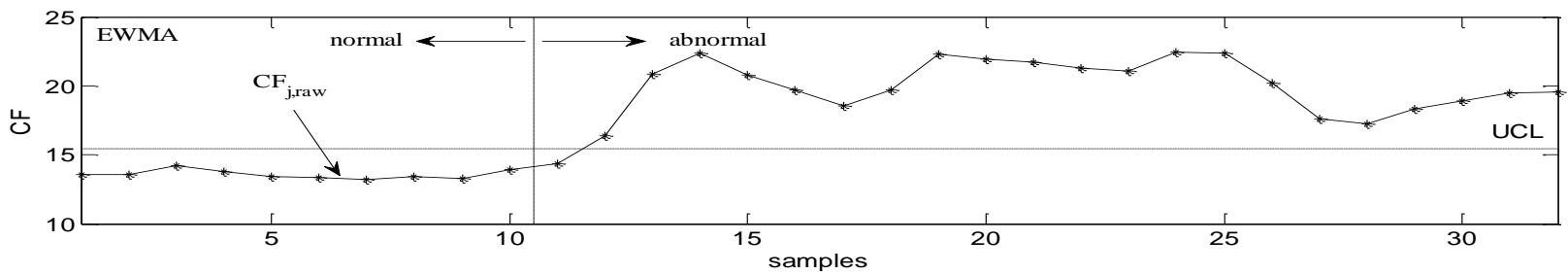

(b)

Fig. 20: Novelty detection of the testing set $S_{2}$ using (a) X-bar and (b) EWMA control charts $(c=0.6)$, trained with $\mathrm{CF}$ values extracted by the raw signals.

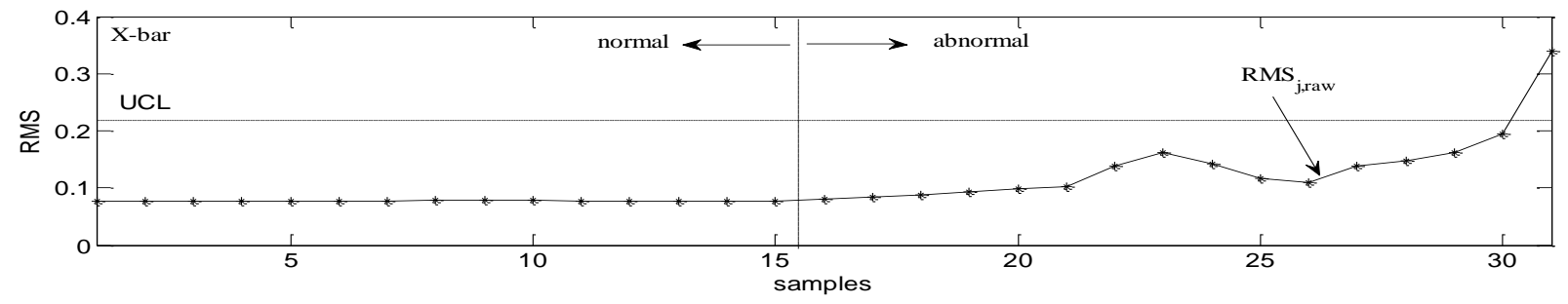

(a)

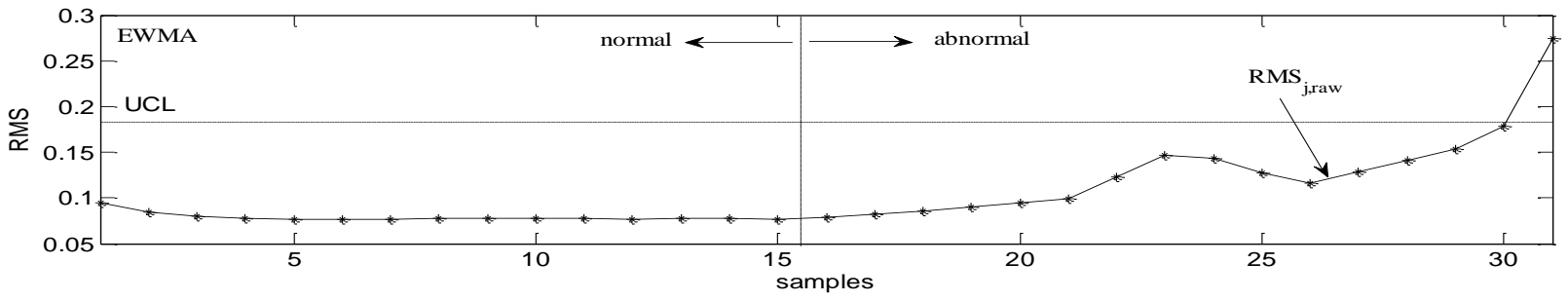

(b)

Fig. 21: Novelty detection of the testing set $S_{1}$ using (a) X-bar and (b) EWMA control charts $(c=0.6)$, trained with RMS values extracted by the raw signals.

Like the proposed hybrid approach, the performance of calculated KU feature extracted by the raw data, is successful in the testing case of data set $S_{2}$, misclassifying as faulty a few normal samples using both the X-bar and EWMA control charts (Fig. 23). On the other hand, as depicted in Fig. 22, the KU abnormal detector does not achieve to focus towards an early abnormal change detection when testing the data set $S_{1}$. Contrary to the RMS features, as shown in Tables 4, 5 and 6, the performance of both statistical models is decreased for both data sets when KU features extracted by data in SMF and ENKU are used.

Furthermore, the SF features detect the abnormal change in the set $S_{1}$ with 6 and 5 testing signals delay 
using the X-bar-based and the EWMA-based process, respectively (Fig. 24). Additionally, Tables 2 and 3 demonstrates that the SF anomaly detector tracks the bearing failure in the set $S_{2}$ only towards the very end. Furthermore as shown, in Tables 5 and 6 , the efficiency of the anomaly detector to track early a fault is improved using features extracted by data in ENKU. Unfortunately, the total performance remains in low levels as a large number of missed detections for faults continue to exist.

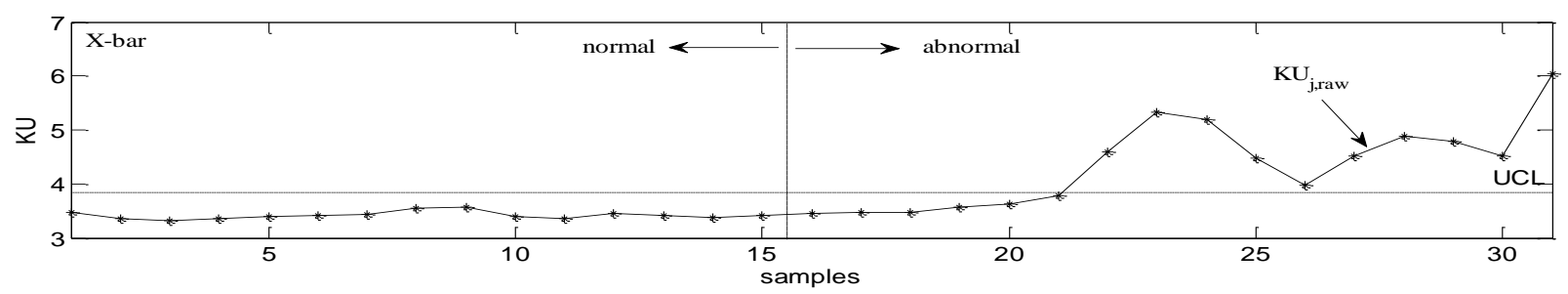

(a)

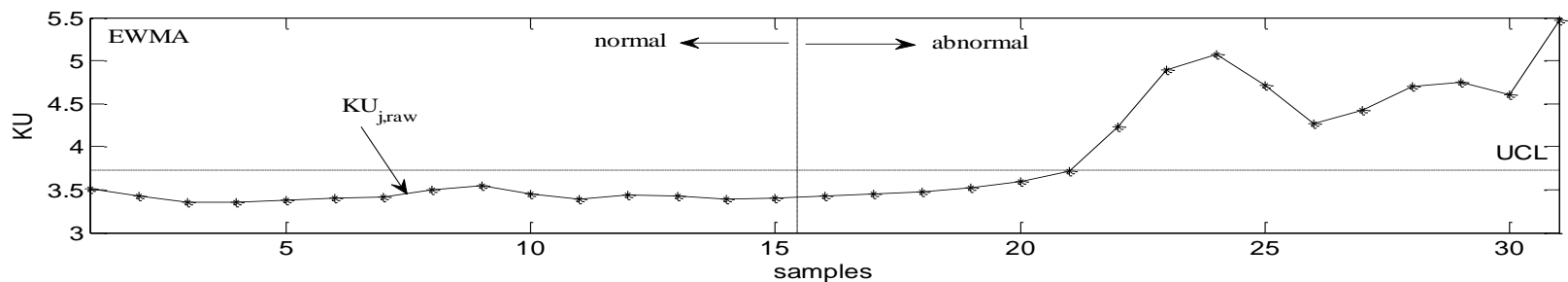

(b)

Fig. 22: Novelty detection of the testing set $S_{1}$ using (a) X-bar and (b) EWMA control charts ( $c=0.6$, trained with $\mathrm{KU}$ values extracted by the raw signals.

Table 2

Performance of the X-bar chart using MSV and other features extracted by raw signals

\begin{tabular}{|c|c|c|c|c|c|c|c|c|c|c|}
\hline & \multicolumn{5}{|l|}{$\mathrm{S}_{1}$} & \multicolumn{5}{|l|}{$\mathrm{S}_{2}$} \\
\hline & MSV & $\mathrm{KU}$ & $\mathrm{CF}$ & SF & RMS & MSV & $\mathrm{KU}$ & $\mathrm{CF}$ & SF & RMS \\
\hline false alarm & 0 & 0 & 0 & 0 & 0 & 1 & 1 & 0 & 0 & 0 \\
\hline missed detection & 1 & 6 & 16 & 7 & 15 & 0 & 0 & 2 & 19 & 18 \\
\hline delay & 1 & 6 & $X$ & 6 & 15 & 0 & 0 & 1 & 19 & 18 \\
\hline accuracy rate $(\%)$ & 96.8 & 80.6 & 48.4 & 77.4 & 51.6 & 96.9 & 96.9 & 93.8 & 40.6 & 43.8 \\
\hline
\end{tabular}

Table 3

Performance of the EWMA chart using MSV and other features extracted by raw signals

\begin{tabular}{|c|c|c|c|c|c|c|c|c|c|c|}
\hline & \multicolumn{5}{|l|}{$\mathrm{S}_{1}$} & \multicolumn{5}{|l|}{$\mathrm{S}_{2}$} \\
\hline & MSV & $\mathrm{KU}$ & $\mathrm{CF}$ & SF & RMS & MSV & $\mathrm{KU}$ & $\mathrm{CF}$ & SF & RMS \\
\hline false alarm & 0 & 0 & 0 & 0 & 0 & 3 & 3 & 0 & 0 & 0 \\
\hline missed detection & 1 & 6 & 16 & 5 & 15 & 0 & 0 & 1 & 19 & 17 \\
\hline delay & 1 & 6 & $X$ & 5 & 15 & 0 & 0 & 1 & 19 & 17 \\
\hline accuracy rate $(\%)$ & 96.8 & 80.6 & 48.4 & 83.9 & 51.6 & 90.6 & 90.6 & 96.9 & 40.6 & 46.9 \\
\hline
\end{tabular}

$X$ : failed fault detection 


\section{Table 4}

Performance of the control charts using KU extracted by shape modified signals

\begin{tabular}{cccccc} 
& \multicolumn{2}{c}{ X-bar } & & EWMA \\
\cline { 2 - 3 } \cline { 5 - 6 } & $\mathrm{S}_{1}$ & $\mathrm{~S}_{2}$ & & $\mathrm{~S}_{1}$ & $\mathrm{~S}_{2}$ \\
\hline false alarm & 0 & 0 & & 0 & 0 \\
missed detection & 16 & 2 & & 16 & 1 \\
delay & $X$ & 1 & & $X$ & 1 \\
accuracy rate $(\%)$ & 48.4 & 93.8 & & 48.4 & 96.9 \\
\hline$X$ : failed fault detection & & & &
\end{tabular}

$X$ : failed fault detection

\section{Table 5}

Performance of the X-bar chart using statistical features extracted by ENKU

\begin{tabular}{|c|c|c|c|c|c|c|c|c|}
\hline & \multicolumn{4}{|l|}{$\mathrm{S}_{1}$} & \multicolumn{4}{|l|}{$\mathrm{S}_{2}$} \\
\hline & KU & $\mathrm{CF}$ & SF & RMS & KU & $\mathrm{CF}$ & SF & RMS \\
\hline false alarm & 0 & 0 & 0 & 0 & 0 & 1 & 1 & 2 \\
\hline missed detection & 12 & 15 & 4 & 13 & 4 & 3 & 13 & 4 \\
\hline delay & 6 & $X$ & 4 & 4 & 1 & 1 & 3 & 0 \\
\hline accuracy rate $(\%)$ & 61.3 & 3.2 & 87.1 & 58.1 & 87.5 & 87.5 & 56.3 & 80.6 \\
\hline
\end{tabular}

Table 6

Performance of the EWMA $(c=0.6)$ chart using statistical features extracted by ENKU

\begin{tabular}{|c|c|c|c|c|c|c|c|c|}
\hline & \multicolumn{4}{|l|}{$\mathrm{S}_{1}$} & \multicolumn{4}{|l|}{$\mathrm{S}_{2}$} \\
\hline & KU & $\mathrm{CF}$ & SF & RMS & KU & $\mathrm{CF}$ & SF & RMS \\
\hline false alarm & 0 & 0 & 0 & 0 & 0 & 2 & 0 & 3 \\
\hline missed detection & 11 & 13 & 4 & 13 & 1 & 1 & 13 & 0 \\
\hline delay & 6 & $X$ & 4 & 4 & 1 & 1 & 3 & 0 \\
\hline accuracy rate $(\%)$ & 64.5 & 58.1 & 87.1 & 59.4 & 96.9 & 90.6 & 59.4 & 90.6 \\
\hline
\end{tabular}

Generally speaking, as it is observed also in the KU abnormal detector (Figs. 22, 23 and 24 and Tables 2, 3 and 4), the RF features give better results compared to their SMF counterparts. As a conclusion, the proposed hybrid approach appears to be a much more consistent and trustworthy process of detecting a possible fault in a bearing. In addition, although the bearing life and failure modes are different from each testing and each bearing, the proposed novel approach still consistently follows the bearing degradation behavior in the whole run-to-failure test.

Moreover, the influence of the weight $c$ on EWMA using KU features is presented in Table 7. As mentioned before, the performance of the EWMA model remains almost unchanged for values of the weight factor $c$ that range between 0.3 and 0.8 . 
Table 7

Performance of the EWMA chart in accordance to the weight factor $c$ using KU extracted by ENKU

\begin{tabular}{ccccccccc}
\hline $\mathrm{c}$ & 0.1 & & 0.3 & & 0.6 & & 0.8 & \\
\hline & $\mathrm{S}_{1}$ & $\mathrm{~S}_{2}$ & $\mathrm{~S}_{1}$ & $\mathrm{~S}_{2}$ & $\mathrm{~S}_{1}$ & $\mathrm{~S}_{2}$ & $\mathrm{~S}_{1}$ & $\mathrm{~S}_{2}$ \\
\cline { 2 - 9 } false alarm & 0 & 0 & 0 & 0 & 0 & 0 & 0 & 0 \\
missed detection & 15 & 2 & 11 & 1 & 11 & 1 & 12 & 2 \\
delay & $X$ & 2 & 10 & 1 & 6 & 1 & 6 & 1 \\
accuracy rate (\%) & 51.6 & 93.8 & 64.5 & 96.9 & 64.5 & 96.9 & 61.3 & 93.8 \\
\hline
\end{tabular}

$X$ : failed fault detection

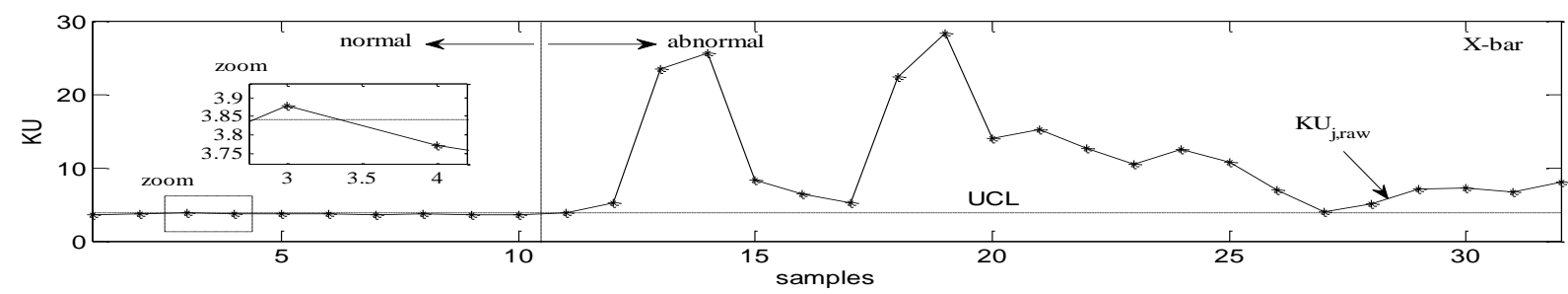

(a)

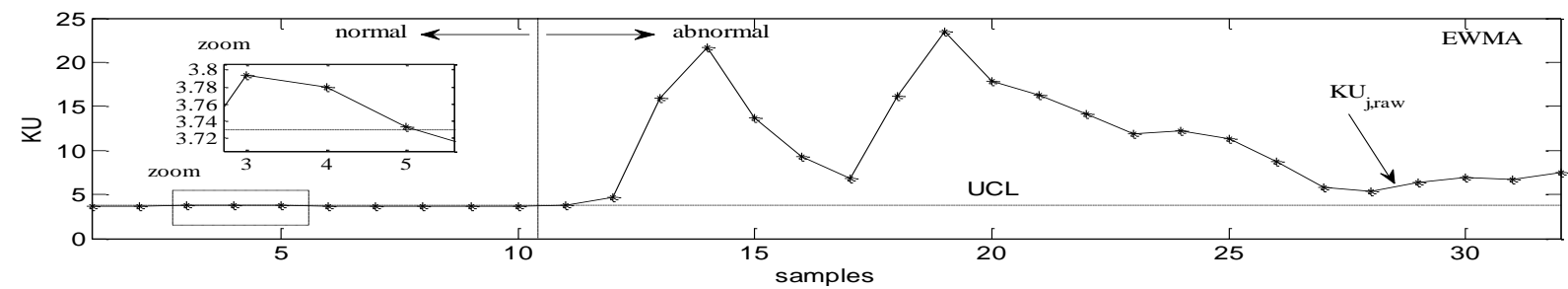

(b)

Fig. 23: Novelty detection of the testing set $S_{2}$ using (a) X-bar and (b) EWMA control charts $(c=0.6)$, trained with $\mathrm{KU}$ values extracted by the raw signals.

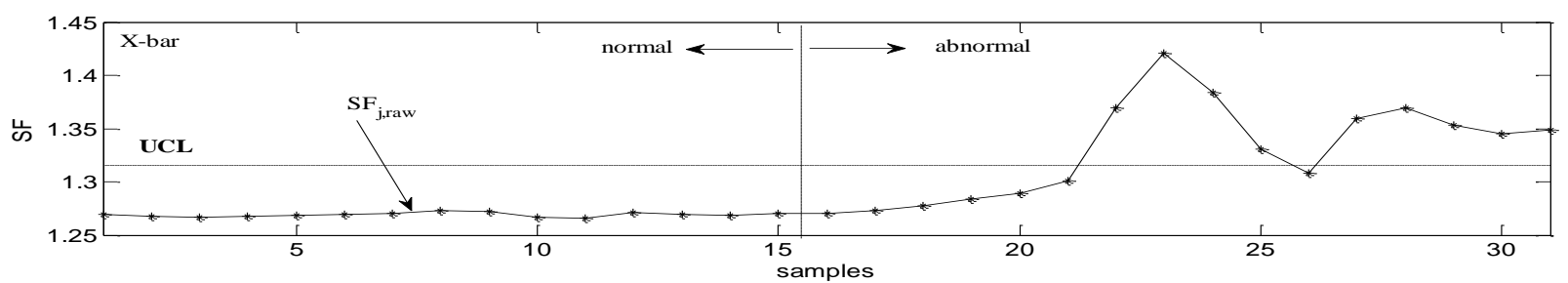

(a)

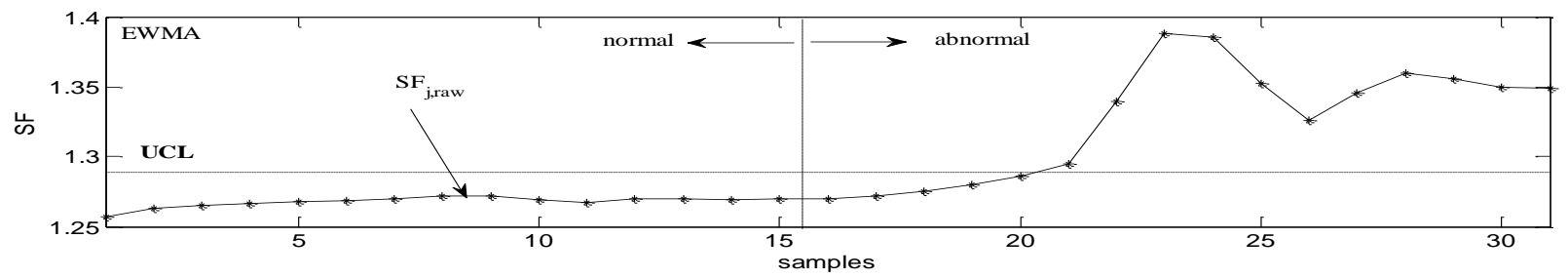

(b)

Fig. 24: Novelty detection of the testing set $S_{1}$ using (a) X-bar and (b) EWMA control charts $(c=0.6)$, trained with SF values extracted by the raw signals. 


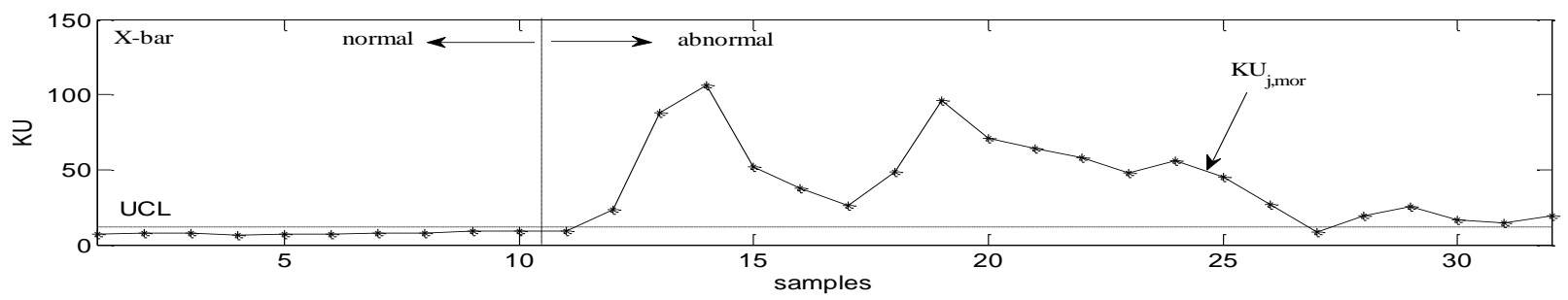

(a)

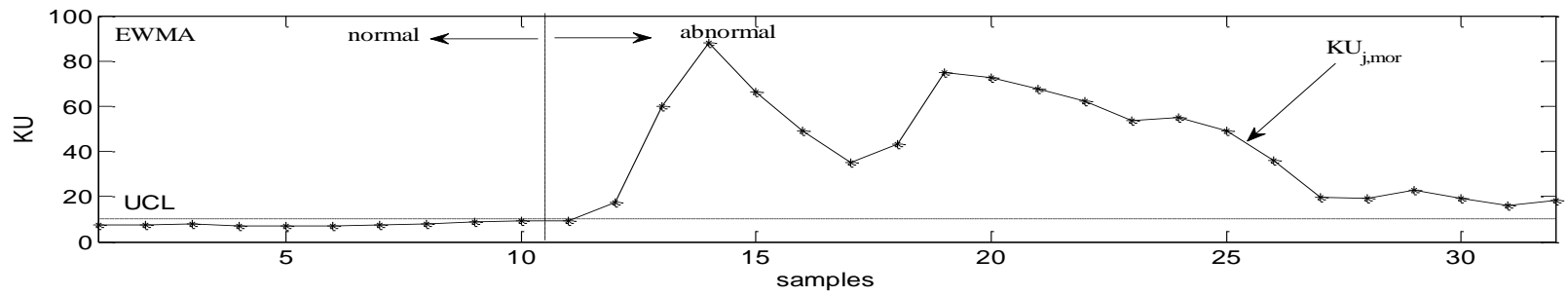

(b)

Fig. 25: Novelty detection of the testing set $S_{2}$ using (a) X-bar and (b) EWMA control charts ( $c=0.6$ ), trained with $\mathrm{KU}$ values extracted by the shape modified signals.

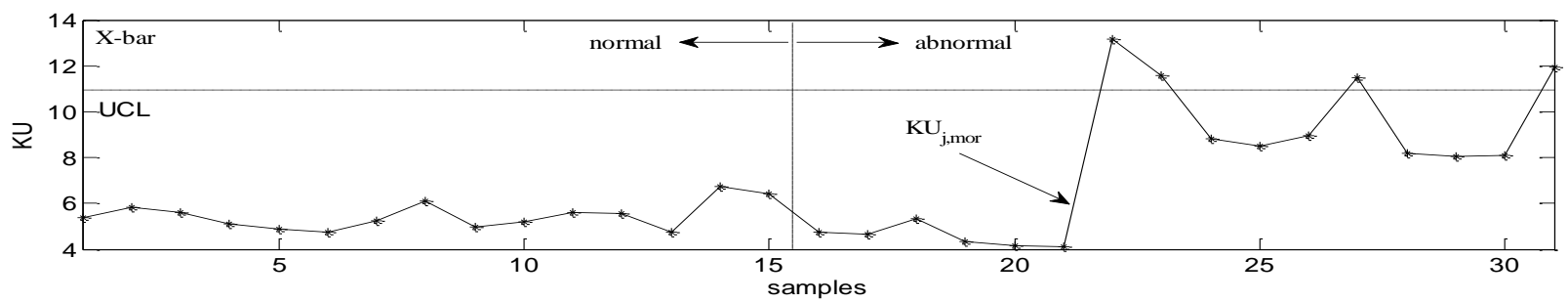

(a)

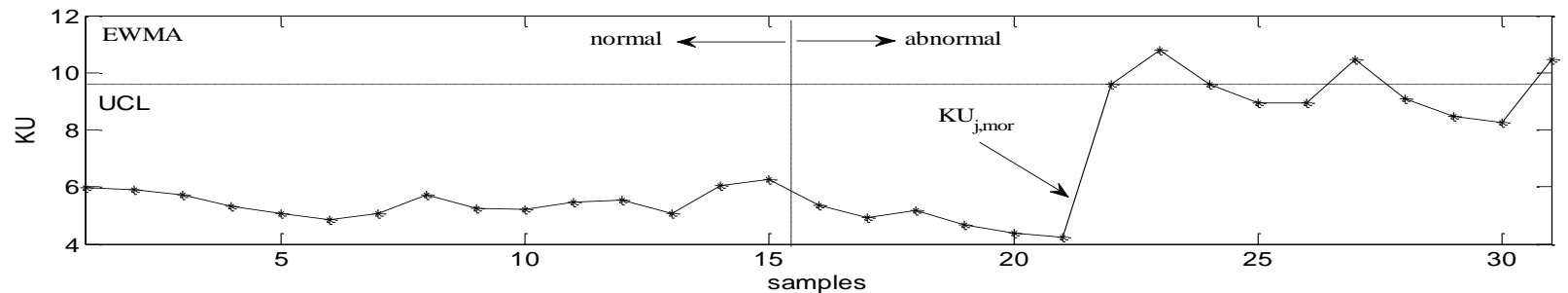

(b)

Fig. 26: Novelty detection of the testing set $S_{1}$ using (a) X-bar and (b) EWMA control charts ( $c=0.6$, trained with $\mathrm{KU}$ values extracted using the kurtogram and envelope analysis.

\section{Conclusion}

Anomaly detection in the framework of fault detection in rotating machinery is a quite challenging task and till now no single model has been verified as the global best one and the diagnostic success mainly depends on the properties of the data handled. In this study an automated hybrid methodology has been proposed, combining the morphological analysis, the Complex Shifted Morlet wavelets and the univariate control charts focusing towards the early and accurate bearing fault detection and identification. The methodology has been applied on two experimental test cases demonstrating successful anomaly detection at the early stages of failures as well as accurate fault classification, presenting low rates of false alarms and missed detections. 


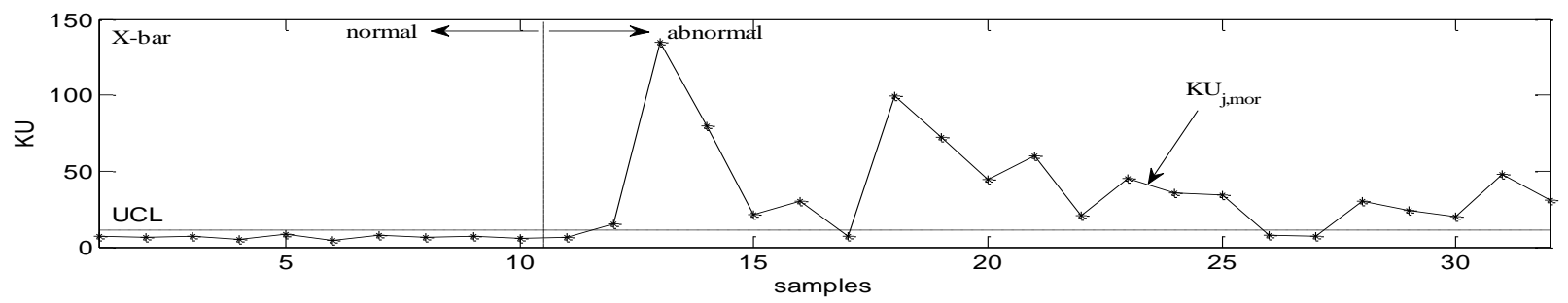

(a)

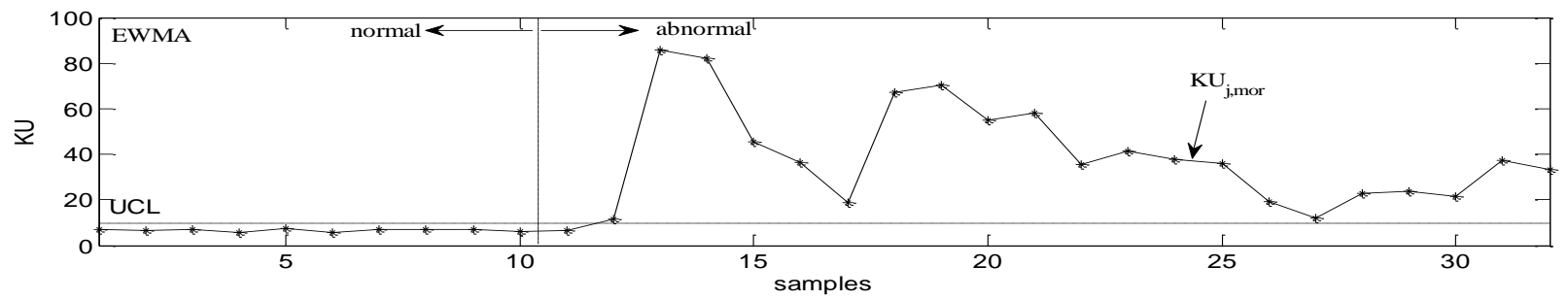

(b)

Fig. 27: Novelty detection of the testing set $S_{2}$ using (a) X-bar and (b) EWMA control charts $(c=0.6)$, trained with $\mathrm{KU}$ values extracted using the kurtogram and envelope analysis.

\section{References}

[1] N. Lu, Z. Xiao, O. P. Malik, Feature extraction using adaptive multiwavelets and synthetic detection index for rotor fault diagnosis of rotating machinery, Mechanical Systems and Signal processing, 52-53 (2015), 393-415.

[2] R. Shao, W. Hu, Y. Wang, X. Qi, The fault feature extraction and classification of gear using principal component analysis and kernel principal components analysis based on the wavelet packet transform, Measurement, 54 (2014), 118-132.

[3] W. Fan, G. Cai, Z. K. Zhu, C. Shen, W. Huang, L. Shang, Sparse representation of transients in wavelet basis and its application in gearbox fault feature extraction, Mechanical Systems and Signal processing, 56-57 (2015), 230-245.

[4] W. He, Y. Zi, B. Chen, F. Wu, Z. He, Automatic fault feature extraction of mechanical anomaly on induction motor bearing using ensemble super-wavelet transform, Mechanical Systems and Signal processing, 54-55 (2015), 457-480.

[5] Z. Li, X. Yan, Z. Tian, C. Yuan, Z. Peng, L. Li, Blind vibration component separation and nonlinear feature extraction applied to the nonstationary vibrations signals for the gearbox multi-fault diagnosis, Measurement, 46 (2013), 259-271.

[6] Y. Qin, Y. Tao, Y. He, B. Tang, Adaptive bistable stochastic resonance and its application in mechanical fault feature extraction, Journal of Sound and Vibration, 333 (2014), 7386-7400.

[7] W. Li, Z. Zhu, F. Jiang, G. Zhou, G. Chen, Fault diagnosis of rotating machinery with a novel statistical feature extraction and evaluation method, Mechanical Systems and Signal processing, 5051 (2015), 414-426.

[8] S. Wang, G. Cai, Z. Zhu, W. Huang, X. Zhang, Transient signal analysis based on LevenbergMarquardt method for fault feature extraction of rotating machines, Mechanical Systems and Signal processing, 54-55 (2015), 16-40. 
[9] M. Kang, J. Kim, Singular value decomposition based feature extraction approaches for classifying faults of induction motors, Mechanical Systems and Signal processing, 41 (2013), 348-356.

[10] B. Muruganatham. M. A. Sanjith, B. Krishnakumar, S. A. V. Satya Mutry, Roller element bearing fault diagnosis using singular spectrum analysis, Mechanical Systems and Signal processing, 35 (2013), 150-166.

[11] F. Cong, J. Chen, G. Dong, F. Zhao, Short-time matrix series based singular value decomposition for rolling bearing fault diagnosis, Mechanical Systems and Signal processing, 34 (2013), 218-230.

[12] B. Kilundu, X. Chiementin, P. Dehombreux, Singular spectrum analysis for bearing defect detection, Journal of Vibration and Acoustics, 133 (2011), 051007-1 - 051007-7.

[13] L. Clifton, D. A. Clifton, Y. Zhang, P. Watkinson, L. Tarassenko, H. Yin, Probabilistic Novelty Detection With Support Vector Machines, Reliability, IEEE Transactions on, 63 (2) (2014), 455467.

[14] V. Jumutc, J. A. K. Suykens, Multi-Class Supervised Novelty Detection, Pattern Analysis and Machine Intelligence, IEEE Transactions on, 36 (2) (2014), 2510-2523.

[15] J. Oster, J. Behar, O. Sayadi, S. Nemati, A. Johnson, G. Clifford, Semi-supervised ECG Ventricular Beat Classification with Novelty Detection Based on Switching Kalman Filters, Biomedical Engineering, IEEE Transactions on, (99) (2015)

[16] T. Heyns, P. S. Heyns, J. P. de Villiers, Combining synchronous averaging with Gaussian mixture model novelty detection scheme for vibration-based condition monitoring of a gear box, Mechanical Systems and Signal Processing, 32 (2012), 200-215.

[17] W. Guo, P. W. Tse, A. Djordjevich, Faulty bearing signal recovery from large noise using a hybrid method based on spectral kurtosis and ensemble empirical mode decomposition, Measurement, 45 (2012), 1308-1322.

[18] S. Wang, J. Yu, E. Lapira, J. Lee, A modified support vector description based novelty detection approach for machinery components, Applied Soft Computing, 13 (2013), 1193-1205.

[19] J. Yu, Bearing performance degradation assessment using locality preserving projections and Gaussian mixture models, Mechanical Systems and Signal Processing, 25 (2011), 2573-2588.

[20] G. Zhang, S. J. Tong, L. Ning, L. Shaoyuan, A modified multivariate EWMA control chart for monitoring process small shifts, International Conference on Modeling, Identification and Control (ICMIC), (2011), 75-80.

[21] C. Zhou, W. Zhang, Recurrence plot based damage detection method by integrating T2 control chart, Entropy, 17 (2015), 2624-2641.

[22] J. Lee, H. Qiu, G. Yu, J. Lin, Rexnord Technical Services, bearing Data Set, IMS, University of Cincinnati, NASA Ames Prognostics Data Repository, NASA Ames, Moffett Field, CA. [Online]. Available: http://ti.arc.nasa.gov/project/prognostic-data-repository.

[23] P. Maragos, W. Schafer, Morphological filters - Part I: their set-theoretic analysis and relations to linear shift-invariant filters, IEEE Transactions on Acoustics, Speech and Signal Processing, 35 (8) (1987), 1153-1169.

[24] N. G. Nikolaou, I. A. Antoniadis, Application of morphological operators as envelope extractors for impulsive-type periodic signals, Mechanical Systems and Signal processing, 17 (6) (2003), 11471162.

[25] L. Zhang, D. Yang, Approach to extracting gear fault feature based on mathematical morphological filtering, Chinese Journal of Mechanical Engineering 43 (2) (2007) 71-75. 
[26] J. Wang, G. Xu, Application of improved morphological filter to the extraction of impulsive attenuation signals, Mechanical Systems and Signal Processing 23 (2009) 236-245.

[27] Y. Dong, M. Liao, X. Zhang, F. Wang, Faults diagnosis of rolling element bearings based on modified morphological method, 25 (4) (2011), 1276-1286.

[28] A. S. Raj, N. Murali, Early classification of bearing faults using morphological operators and fuzzy inference, IEEE Transactions on Industrial Electronics, 60 (2) (2013), 567-574.

[29] D. Wang, P. W. Tse, Y. L. Tse, A morphogram with the optimal selection of parameters used in morphological analysis for enhancing the ability in bearing fault diagnosis, measurement Science and Technology, 23 (2012), 065001.

[30] I. A. Antoniadis, C. T. Yiakopoulos, K. C. Gryllias, K. I. Rodopoulos, IFESIS: Instantaneous Frequency Estimation via Subspace Invariance properties of wavelet Structures, Mechanical Systems and Signal Processing, 49 (2014), 264-284.

[31] T. P. Le, P. Argoul, Continuous wavelet transform for modal identification using free decay response, Journal of Sound and Vibration, 277 (1) (2004), 73-100.

[32] N. G. Nikolaou, I. A. Antoniadis, Demodulation of vibration signals generated by defects in rolling element bearings using complex shifted Morlet wavelets, Mechanical Systems and Signal Processing, 16 (2002), 677-694.

[33] K. C. Gryllias, I. A. Antoniadis, Estimation of the instantaneous rotation speed using complex shifted Morlet wavelets, Mechanical Systems and Signal Processing, 38 (1) (2013), 78-95.

[34] R. Roy, A. Paulraj, T. Kailath, ESPRIT - a subspace rotation approach to estimation of parameters of cissoids in noise, IEEE Transactions on Acoustics, Speech and Signal Processing, 34 (1986), 1340-1342.

[35] H. M. Hanson, P. Maragos, A. Potamianos, A system for finding speech formants and modulation via energy separation, IEEE Transactions on Speech and Audio Processing, 2 (3) (1994), 436-442.

[36] C. W. Kang, P. H. Kvam, Basic Statistical Tools for Improving Quality, Wiley, (2011)

[37] N. Abbas, M. Riaz, R. J. M. M. Does, An EWMA-Type Control Chart for Monitoring the Process Mean Using Auxiliary Information, Communications in Statistics - Theory and Methods, 43 (2014), 3485-3498.

[38] C. T. Yiakopoulos, K. C. Gryllias, I. A. Antoniadis, Spectral effects of the slip motion in defective rolling element bearings, Advances in Vibration Engineering, 4 (2) (2005), 199-211. 